\title{
Thermal Energy Process Heat for Lunar ISRU: Technical Challenges and Technology Opportunities
}

\author{
Pierce E. C. Gordon ${ }^{1}$ \\ The University of Michigan, Ann Arbor, MI, 48109, USA \\ Anthony J. Colozza ${ }^{2}$ \\ QinetiQ NA, Cleveland, OH, 44135, USA \\ Aloysius F. Hepp ${ }^{3}$ \\ NASA Glenn Research Center, Cleveland, OH, 44135, USA \\ Richard S. Heller ${ }^{4}$ \\ Massachusetts Institute of Technology, Cambridge, MA, 02139, USA \\ Robert Gustafson ${ }^{5}$ \\ Orbital Technologies Corporation (ORBITEC), Madison, WI, 53717, USA \\ Ted Stern ${ }^{6}$ \\ DR Technologies, Inc., San Diego, CA, 92126, USA \\ and \\ Takashi Nakamura $^{7}$ \\ Physical Sciences, Inc., Pleasanton, CA, 94588, USA
}

\begin{abstract}
Oxygen production from lunar raw materials is critical for sustaining a manned lunar base but is very power intensive. Solar concentrators are a well-researched and developed technology for harnessing the sun's energy to heat regolith to high temperatures (over 1273K). The high temperature and potential material incompatibilities present numerous technical challenges. This study compares and contrasts different solar concentrator designs that have been developed, such as Cassegrains, offset parabolas, compound parabolic concentrators, and secondary concentrators. Differences between concentrators made from lenses and mirrors, and differences between rigid and flexible concentrators are also compared. Possible substrate elements for a rigid mirror concentrator are chosen and discussed, with the following criteria: low CTE, high modulus of elasticity, and low density. Possible lunar locations for concentrator technology are also compared and contrasted. Several environmental and processing-related challenges related to dust and optical surfaces are addressed. This paper also examines the various sources of thermal energy that can be utilized for ISRU applications on the lunar surface. These include heat from nuclear and electric sources and solar concentrators. The options for collecting and transporting the heat to the processing reactor for each source are examined. The overall system requirements for each thermal source are compared and the system limitations, such as maximum achievable temperature are discussed.
\end{abstract}

\footnotetext{
${ }^{1}$ Summer Intern, RPC, NASA Glenn Research Center, Department of Mechanical Engineering.

${ }^{2}$ Research Engineer, QinetiQ NA/NASA Glenn Research Center, 21000 Brookpark Rd. MS 309-1.

${ }^{3}$ Principal Investigator, Senior Scientist, NASA Glenn Research Center, REB, 21000 Brookpark Rd., MS 302-1.

${ }^{4}$ NASA Graduate Student Researcher, Department of Mechanical Engineering, 77 Mass. Ave., Room 3-173.

${ }^{5}$ Principal Aerospace Engineer, 1212 Fourier Drive, AIAA member.

${ }^{6}$ VP/General Manager, Space Power, Optics and Thermal Products, 9431 Dowdy Dr., AIAA member.

${ }^{7}$ Area Manager, Space Exploration Technologies, 6652 Owens Drive, AIAA member.
} 


\section{Nomenclature}

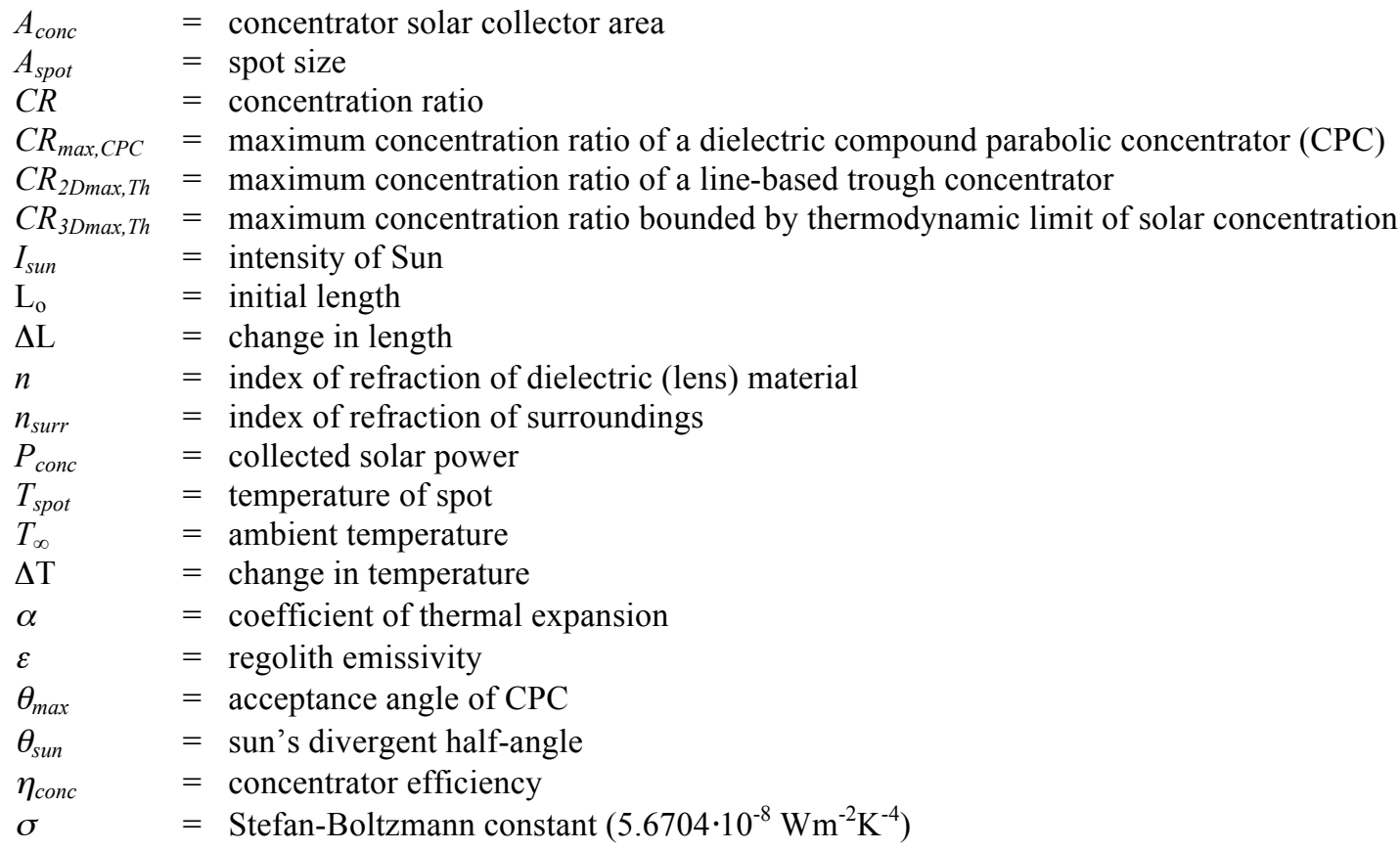

\section{Background}

Colar concentrators use optical media, such as lenses and mirrors, to focus incident (solar) light. The concentrated light can then be focused to produce thermal or electrical energy in conjunction with photovoltaic (PV) cells. Solar concentrator technologies date back to Leonardo da Vinci, who suggested making a parabolic mirror four miles across in 1515 as a means of melting metals for industrial processes ${ }^{1}$. Solar concentrators have been applied in industry, consumer products, and numerous advanced technologies. Concentrator technologies have applications across the spectrum; paraboloid shaped antennae dishes used for satellite communications use similar geometries for radio waves that mirror-based concentrators use for visible light. Parabolic trough technologies are applied for cutting-edge terrestrial solar power plants and in space via a Stretched-Lens Array (SLA) Fresnel concentrator design. ${ }^{2}$ Concentrator technologies for PV boast a higher specific power rating than planar PV without concentrators; the promise of higher efficiencies and higher power output for lower mass and cost are enticing goals spurring further technological developments.

\section{A. Introduction to Solar Concentrators}

A solar concentrator collects solar radiation and concentrates the energy into a reduced spot of light. This concentrated sunlight can be utilized to heat up a material, such as the lunar regolith. The surface temperature of the material at the spot of concentrated sunlight is governed by an energy balance between the power flux density of the concentrated sunlight and the heat losses to the surroundings. The heat losses will be a combination of:

- Conduction to the surrounding material

- Convection to the atmosphere or gas

- Radiation to the surroundings.

If the material is within a vacuum, as on the lunar surface and has a low thermal conductivity, as with the lunar regolith, then the conduction and convection methods of heat transfer can be ignored. Therefore radiation heat transfer, as expressed by the Stefan-Boltzmann Law (given in Eq. (1)) will determine the ultimate achievable material temperature within the illuminated spot.

$$
P_{\text {conc }}=\sigma \varepsilon A_{\text {spot }}\left(T_{\text {spot }}^{4}-T_{\infty}^{4}\right)
$$

The concentrator power, $\mathrm{P}_{\text {conc }}$ is determined by solar intensity, $I_{\text {sun }}$, assumed to be the average solar intensity of $1353 \mathrm{~W} / \mathrm{m}^{2}$, the area of the concentrator and the concentrator efficiency ${ }^{3}, \eta_{\text {con }}$, as given in Eq. (2). For the lunar surface the temperature of the surroundings $T_{\infty}$, is assumed to be $270 \mathrm{~K}$. It should be noted that the exact ambient

2

American Institute of Aeronautics and Astronautics 
temperature is wholly dependent on lunar surroundings, and can only be calculated through a precise energy balance of the desired operation geography and sun angle. Due to the radiation losses of the heated material, as the spot size increases there is a decrease in the achievable steady state material temperature within the illuminated spot.

$$
P_{\text {conv }}=\eta_{\text {conc }} A_{\text {conc }} I_{\text {sun }}
$$

This relationship between the solar flux captured by the concentrator and the concentrated spot size is expressed as the concentration ratio (CR) of the concentrator. It is determined by equating Eq. (1) and Eq. (2) and solving for the ratio of concentrator area over spot area $\left(\mathrm{A}_{\mathrm{con}} / \mathrm{A}_{\mathrm{spot}}\right)$. This ratio is given by Eq. (3).

$$
C R=\frac{\varepsilon \sigma\left(T_{\text {spot }}^{4}-T_{\infty}^{4}\right)}{I_{\text {sun }} \eta_{\text {conc }}}
$$

There is a theoretical limit on the concentration ratio for concentrating sunlight. This is due to the fact that the sun is not a point source of light. The sun's disk has a half angle of $4.653 \mathrm{mrad}\left(\theta_{\mathrm{s}}\right)$ when viewed from Earth's distance. The theoretical limit on concentration ratio is given by 1 over the sine squared of the solar half angle ${ }^{39}$, as expressed in Eq. (4) $)^{1}$.

$$
C R_{\max }=\frac{1}{\sin ^{2}\left(\theta_{s}\right)}=\frac{1}{\sin ^{2}(.004653)}=46,188
$$

The relation between the regolith temperature within the spot of concentrated sunlight and the concentrator concentration ratio at differing emissivities is displayed in Fig. 1. For processing materials on the lunar surface, temperatures up to the melting point of the lunar regolith are of interest. Because the regolith is a mixture of materials it melts over a temperature range of $1373 \mathrm{~K}$ to 1653 $\mathrm{K}^{40}$. The corresponding $\mathrm{CRs}$ needed to achieve these temperatures are approximately 150 and 320 respectively. When other losses of the system are considered, such as reflectivity of the mirrors, end angle losses, losses due to heat pipe or optical cable use, and the deterioration of mirror performance due to lunar environmental effects, the required $\mathrm{CR}$ becomes much higher. The maximum CR of each concentrator is limited by the overall system design as well other

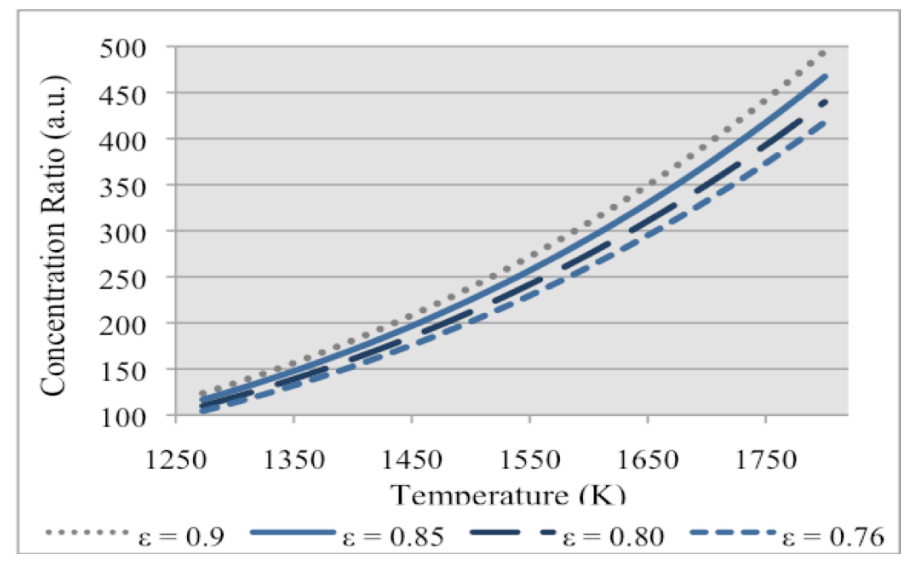

Figure 1. Minimal Concentration Ratio versus temperature model at different emissivity levels of a solar concentrator. The sink temperature was estimated to be $270 \mathrm{~K}$, but actual lunar sink temperatures are dependent on lunar energy balance. Graph only considers radiation heat transfer from surface. specific limitations that are discussed below.

While it may seem logical that a larger concentrator, capable of capturing more solar energy, can produce a higher spot temperature this is not the case because as the concentrator size increases so does the spot size as given by the concentration ratio expressed in Eq. (2). This larger spot size in turn increases the heat losses to the surroundings, thereby limiting the spot temperature. This observation implies an important consideration about solar concentrators; a large solar flux does not mean a higher temperature. More often than not, with larger concentrators, their CRs are lower due to larger shape errors; thus, the spot temperatures are less likely to reach the high values necessary for oxygen production. This does not mean, however, that the aim is always to forego capturing less solar energy for a higher CR; a certain amount of thermal energy must be captured to reach set goals for the desired material process such as oxygen production. For example, a preliminary study previously performed determined that $15,241 \mathrm{~W}$ of thermal power was required to produce $1000 \mathrm{~kg} /$ year of oxygen from the hydrogen 
reduction of ilmenite process ${ }^{3}$ It is the combination of the maximum achievable temperature as well as the total thermal power supplied that dictates the sizing and required applicability of a concentrator design to a specific task.

Some of the characteristics desired in a concentrator for a lunar surface mission, such as large size, low mass, low cost and deployability, tend to limit the achievable concentration ratio and therefore limit the process temperature. One method to enhance the concentrator concentration ratio is to utilize a secondary and possibly tertiary concentrator within the system. This type of staged concentration of sunlight provides some key benefits to the concentrator system. It allows the geometry tolerances of the primary concentrator to be relaxed which makes stowage and deployment easier. The secondary concentrator can then be made highly precise to accurately take the concentrated light from the primary concentrator and focus it down further to achieve the desired concentration ratio. Another benefit of staging the concentration of the sunlight is that the light can be redirected to the desired process location as it is being further concentrated. Utilizing a secondary and possibly a tertiary concentrator within the system can enable the primary concentrator to track the sun while keeping the concentrated sunlight focused on a specific location relative to the surface. The downside to utilizing multiple concentrators is that it will decrease the overall efficiency of the system. There are losses associated with each mirror or lens utilized within the concentrator system. These losses are due to geometry inaccuracies, light absorption and scattering. The following items help minimize these effects:

- Short ray length, which minimizes the effect of mirror errors.

- For mirrors, high reflectivity, specularity and minimal geometrical errors.

- For lenses, high transmissibility, and minimal losses from groove shadowing or spectral shifting.

\section{B. The Application of Concentrators to Lunar Oxygen Extraction}

Concentrators have been extensively researched as a key technology for In-Situ Resource Utilization (ISRU) research and development, specifically for the production of oxygen from lunar regolith. Oxygen obtained from the regolith could be used for extended human habitation on the moon, rocket propellant, or for fuel cell technology. Lunar soil is approximately $45 \%$ oxygen by weight, but it is chemically locked up in oxide compounds with silicon, magnesium, titanium, and other elements. Table 1 below shows the major mineral compositions in the highland and mare regions of the moon. ${ }^{4}$

To obtain the oxygen, electrochemical processes have been developed which can require temperatures ranging from 1273-1800 K for extended periods of time. The limiting temperatures of this range refer to two specific processes, the hydrogen reduction process and the carbothermal reduction process respectively. The carbothermal and hydrogen reduction processes are two of over 20 such processes for extracting lunar oxygen. We not consider the other process, but some examples are listed for reference: ${ }^{4-6}$

- Ilmenite Reduction by Carbon Monoxide

- Ilmenite Reduction with Methane

- Glass Reduction with Hydrogen

- Reduction with Hydrogen Sulfide

- Extraction with Fluorine

- Carbochlorination

- Chlorine Plasma Reduction

- Molten Silicate Electrolysis

- Fluxed Molten Silicate Electrolysis

- Caustic Solution and Electrolysis

- Magma Partial Oxidation

- Li or Na Reduction

- Vapor Phase Reduction

- Ion (Plasma) Separation

- Plasma Reduction of Ilmenite

- HF Acid Dissolution

- $\mathrm{H}_{2} \mathrm{SO}_{4}$ Acid Dissolution

- Hydrogen/Helium/Water Production from Soil
Table 1. Lunar Surface Mineral Compositions

\begin{tabular}{|c|c|c|}
\hline Compound & $\begin{array}{c}\text { Highland } \\
\text { (weight \%) }\end{array}$ & $\begin{array}{c}\text { Mare } \\
\text { (weight \%) }\end{array}$ \\
\hline $\mathrm{SiO}_{2}$ & 44.5 & 41.0 \\
\hline $\mathrm{Al}_{2} \mathrm{O}_{3}$ & 26.0 & 12.8 \\
\hline $\mathrm{FeO}$ & 5.77 & 16.2 \\
\hline $\mathrm{CaO}$ & 14.9 & 12.4 \\
\hline $\mathrm{MgO}$ & 8.05 & 9.2 \\
\hline $\mathrm{TiO}_{2}$ & ---- & 7.3 \\
\hline
\end{tabular}

4

American Institute of Aeronautics and Astronautics 


\section{Mirror Design Considerations}

Different concentrator geometries have advantages and disadvantages depending on the application. As such, each type of design would be used for different task or environment. In the following section, an overview of mirror geometry specifics, advantages and disadvantages, possible design opportunities, and design comparisons are discussed. A chart with literature values relating concentrator types and maximum CR is shown in Table 2..$^{7-11}$

\section{A. Cassegrain Reflectors}

A Cassegrain reflector, shown in Fig. 2, is a reflecting-mirror assembly where incident solar light is focused by a collection of two or more mirrors towards a desired focus behind the primary mirror. The primary concave mirror focuses incident light in the direction of the light source, and the secondary convex mirror refocuses that light towards a hole in the primary mirror, as illustrated in Fig. 3. The technology is used primarily with telescopes; both the Hubble Space Telescope and its replacement, the James Webb Space Telescope, are Cassegrain assemblies. ${ }^{12}$

The types of Cassegrains differ normally in shape of primary and secondary mirrors. For telescopes, known as imaging devices, most differentiations are used to counteract effects of coma, spherical, and chromatic aberrations; in most cases, the more aspheric the design and the more accurate the desired geometry, the more complex and costly the manufacturing process. A possibility for reducing the cost would be to use a primary spherical mirror and an aspheric secondary mirror to correct aberrations. Personnel at Physical Sciences Incorporated (PSI) conducted simple ray-tracing analyses of the difference between two configurations; one with two spherical mirrors, and one with a spherical primary and aspherical secondary, and found the aspherical secondary sufficiently corrects spherical aberration for their purposes. $^{13}$

Because a Cassegrain's secondary focus can be positioned just behind the primary mirror, the ray length of the mirror is usually much smaller than that of other mirror geometries of the same size. Thus, scattering effects caused by mirror aberrations are less likely to cause a decrease in spot size or lower solar flux efficiency. Rigid Cassegrains are usually the concentrator assemblies with the highest CRs of any concentrator design.

Table 2. Approximation of concentrator type and maximum Concentration Ratio (CR)

\begin{tabular}{|c|c|}
\hline Concentrator Type & $\begin{array}{c}\text { Concentration Ratio } \\
\text { Range }\end{array}$ \\
\hline Fixed Cassegrain Concentrator & 8000 max. \\
\hline Offset Parabola & 4000 max. \\
\hline $\begin{array}{c}\text { Compound Parabolic } \\
\text { Concentrators (CPC) }\end{array}$ & $\begin{array}{c}6.5-10 \text { (line-based), } 81 \\
\text { (spot-based) }\end{array}$ \\
\hline Inflatable Offset & 2400 \\
\hline Trough (Line-focus) & $8-30$ \\
\hline
\end{tabular}

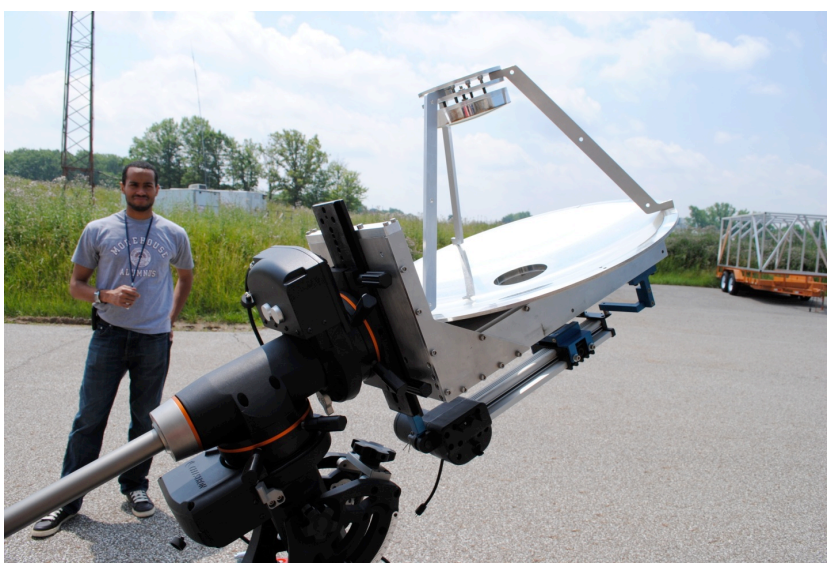

Figure 2. Pierce Gordon standing next to a Cassegrain concentrator manufactured for NASA GRC.
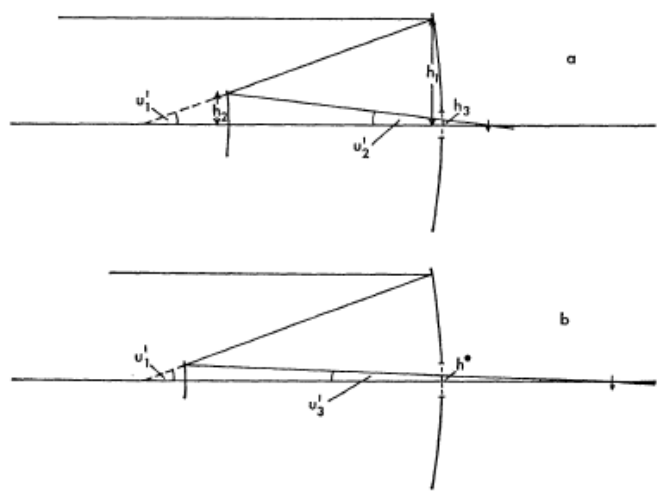

Figure 3. Cassegrainian Light Ray Path.

However, setting up a complex array of Cassegrains, or a particularly large single Cassegrain mirror in a lunar environment, is still a complex engineering task involving a considerable design effort. The main issue with utilizing a Cassegrain concentrator, or any geometry concentrator, is the packaging and deployment. One possible 
folding maneuver is the one used by the James Webb Space Telescope, where the edges of the telescope fold back like leaves of a table during launch and deployment, and spread back into place during operation. ${ }^{12}$ Other deployment possibilities are still available and are being researched. Also, arrays with multiple concentrators have demonstrated to be very plausible; as evidenced from concentrator assemblies manufactured by PSI, Cassegrain assemblies are highly modular, and Cassegrain concentrator arrays are a very plausible technology option. ${ }^{13}$

Although the theoretical performance of a Cassegrain concentrator can be very high, there are several issues with Cassegrain technology that could impact their use. They must be capable of tracking the sun very accurately to work properly; because of its high CR; if the solar energy was no longer parallel with the mirror's normal, the spot could focus onto the primary mirror instead of into the mirror cavity, which could cause catastrophic mirror damage. Most Cassegrain designs also have supports that hold the secondary mirror into place which blocks some solar energy from ever reaching the first mirror; thus the entire mirror is not used to its full potential. Because Cassegrains have two mirrors, they have two sources of reflectivity loss. Though they can be coated with highreflective materials and transparent resistant films to limit losses, the added energy loss and possibility for deterioration due to oxidation of reflective films still must be considered. Because the Cassegrain's focus moves along with the tracking mirror, a process is needed to transfer the solar energy to the stationary reactor; each energy transfer option has inherent heat losses. Though the design has disadvantages, Cassegrain technologies are the most modular and applicable individual concentrator technology for thermal concentration opportunities. The same Cassegrain technology can be efficiently developed for most lunar environments.

\section{B. Offset Parabola}

One misgiving introductory optics courses typically give is that the most effective focusing geometry of parallel light rays is one of a circular cross section. In fact, circles create a slight aberration of light rays at the focus; the best geometry is in fact, a parabola. Though the sun's rays diverge at a small angle, and some concentrators do account for such aberration, parabolic geometries are sufficient approximations for optimum focusing technology. Parallel light rays converge at the parabola's focus. It is, in fact, why most advanced non-imaging optics technologies utilize a parabola as the cornerstone of the geometrical design. The offset parabola configuration, then, is a reflecting mirror whose shape represents a portion of a whole paraboloid. If the Cassegrain primary mirror is a paraboloid located at the center, an offset parabola is a portion of the paraboloid's sidewall, as seen in Figs 4 and $5 .{ }^{14}$

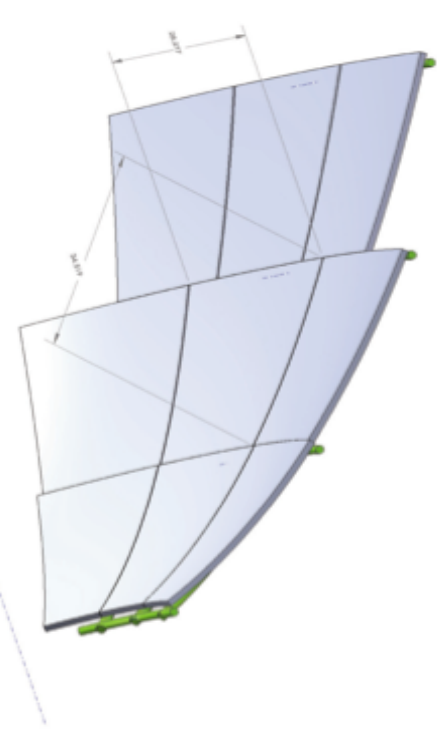

Figure 4. Concentrator with Off-Set Parabolaic Geometry.

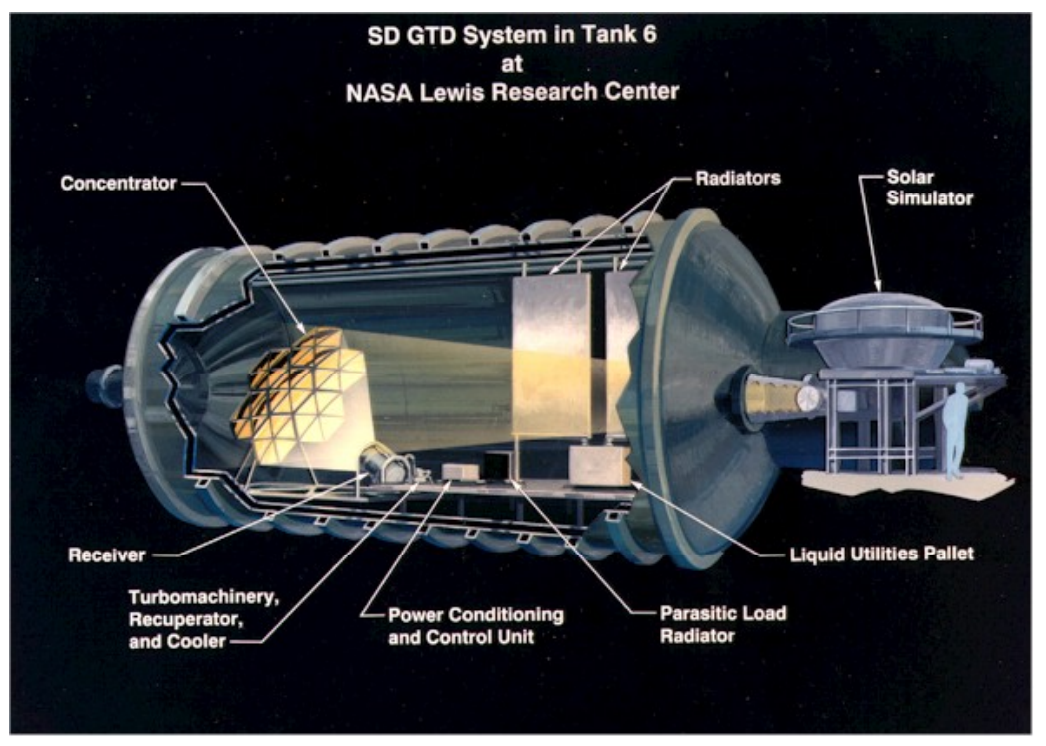

Figure 5. Diagram of Ground Test Demonstrator at NASA GRC using an offset parabolic solar concentrator.

and below the concentrator, the need for a secondary concentrator can be eliminated. Therefore the reflected light, fortunately, only has to reflect once before it reaches its focus, thus decreasing reflectivity losses. The ability to design the concentrator with the focus on or near the surface provides a significant benefit in integrating this concentrator geometry into an oxygen reactor design. Because currently designed reactor models are immobile, the 
solar flux can be easily pointed towards a window in the reactor, directly illuminating the regolith. This configuration would work most effectively at the moon's poles, like the lunar South Pole, where the moon is nearly in constant illumination by the sun. ${ }^{4}$ In fact, the spot could be focused in any direction perpendicular to the direction of incident sunlight, e.g. to the side or above the concentrator. This opens up the possibility for ingenious design opportunities for optical steering by secondary concentrators.

Another advantage to this geometry is that the parabolic mirror can be configured so its entire mirror surface is sunlit, unlike Cassegrains, where some of the sunlight is blocked by structure necessary to keep the secondary mirror in position. Because the parabola is essentially a small section of a much larger paraboloid mirror, the ray lengths of the offset parabola will always be longer than those of a similarly sized Cassegrain mirror. Thus, offset parabolic mirrors will either always have lower CRs, or smaller captured solar flux, than Cassegrains. A mirror design like an offset parabola, however, has more technology opportunities for application. The geometry lends itself to a number of applications such as illuminating the surface to provide 'thermal wadis' to store thermal energy to protect rovers and other assets from harsh thermal cycling effects. ${ }^{15}$

\section{Compound Parabolic Concentrators (CPCs)}

CPCs are concentrators geometrically designed to capture light at a much larger acceptance angle than other concentrators, lessening the need for highly accurate tracking capabilities. Spot-based CPCs both have the cross section of two deep parabolas; the edge on one parabola is the focus of the other, and vice versa. The geometry is rotated about the axis of concentration, and a spot focus manifests on the other side.

Tracking does not need to be as precise or accurate with these concentrators, because the concentrator accepts light within approximately $3^{\circ}$ of their maximum acceptance angle, depending on its design. ${ }^{16}$ For a constantly moving sun with a possible incidence angle range of 90

degrees, however, use of a CPC as a primary concentrator would still require some degree of tracking. CPCs are manufactured as both dielectrics (lenses), or as hollowed mirrors. Examples of each type are shown in Figs. 6 and 7 respectively. The largest theoretical CR of all CPC's, dielectric and mirror-based, is defined by Eq. (5): ${ }^{16}$

$$
C R=\frac{n^{2}}{\sin ^{2}\left(\theta_{\max }\right)}
$$

In a mirror-based CPC, the refractive index $n$ is assumed to be 1 . As shown from Eq. (5), the CR is inversely proportional to the maximum acceptance angle. This means larger concentration CPCs have a small outer diameter and a long length. Consider, then, a CPC used as the primary concentrator; the diameter of the $\mathrm{CPC}$ would need to be around the same length as the diameter of the solar flux. This would mean the length of the CPC would be considerably long; both the volume and weight of such an instrument are not feasible for use as a primary concentrator.

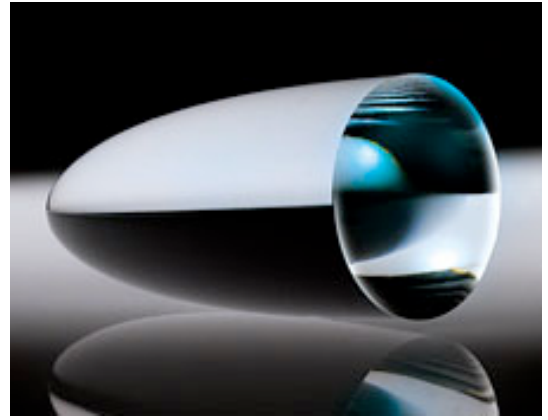

Figure 6. Hollow-Mirror CPC.

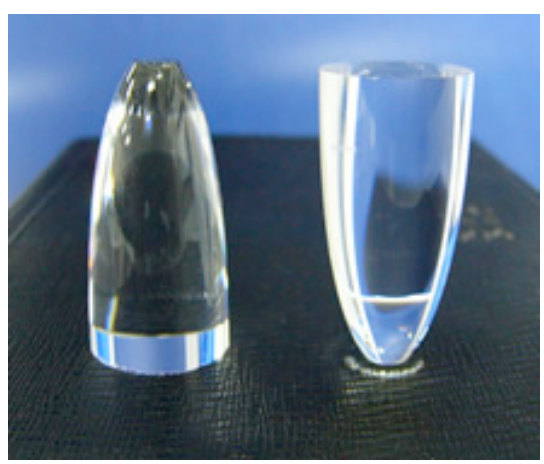

Figure 7. Lens CPC.

Dielectric CPCs are also limited by another restriction; the propagating light is assumed to be totally internally reflected by the concentrator walls. The maximum concentration due to this restriction is shown by Eq. $6:{ }^{16}$

$$
C R_{\max , C P C}=\frac{1}{\left(1-\frac{2}{n^{2}}\right)^{2}}
$$

This equates to a maximum concentration of $C R_{\max , C P C}$ of 81 for an example refractive index of $n=1.5$ for crown glass. ${ }^{9}$ Such concentrations are too small for achieving temperatures necessary for melting lunar regolith by itself. However, there is still a possibility for CPCs to be used as secondary concentrators. 


\section{Trough (Line-focus) designs}

As discussed previously the most common concentrator shape is a parabolic dish, or cone in which the incident light is directed towards a spot focus of a specified area. Another approach is to utilize a trough or cylindrical configuration, which sweeps out the parabola along a line perpendicular to its two-dimensional shape. The incident light will then focus onto a line. Two main types of line-focus concentrators have been developed; one, well known for its terrestrial applications, is the parabolic trough. This technology is used in large-scale solar plant designs, and can also be adapted for use in water purification. Another line-focus technology developed in recent years, the Stretched Lens Array (SLA), uses stretched space-rated silicon as a specifically shaped lens to direct light towards a desired focus as displayed in Fig. 8. ${ }^{2}$ The SLA technology offers state-of-the-art performance in the following metrics: areal power density $\left(\mathrm{W} / \mathrm{m}^{2}\right)$, specific power $(\mathrm{W} / \mathrm{kg})$, stowed power density $\left(\mathrm{kW} / \mathrm{m}^{3}\right)$, operating voltage $(\mathrm{V})$, and array power capacity ( $\mathrm{kW}$ to multi-MW). ${ }^{2}$

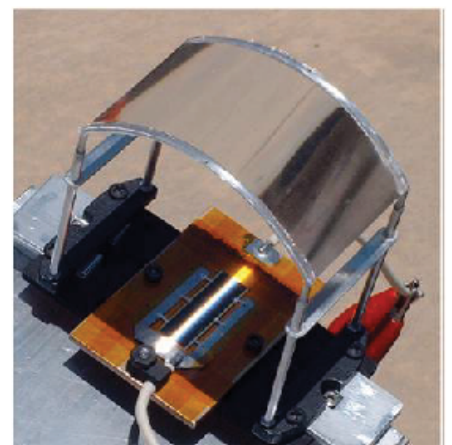

Figure 8. The Stretched-Lens Array focuscing incident light onto PV collector. The technology can be manufactured using a roll-to-roll process, making it easy to rapidly mass-produce any desired quantity. ${ }^{8}$

However, in thermal applications where high temperatures are necessary for processes such as oxygen production, the line-focus design falls short. The maximum possible CR of a line concentrator, as bounded by the thermodynamic limit for solar concentration, ${ }^{1}$ is described by the relationship given by Eq. 7:

$$
C R_{2 D \max , T h}=\frac{1}{\sin \theta_{\text {sun }}}
$$

Because the half-angle of the sun's incident light $\theta_{\text {sun }}$ is $0.275^{\circ}$, the maximum concentration of a linear, or trough concentrator, as defined by its thermodynamic limit is approximately 208 , and realistic linear concentrators aim for a maximum CR of about $30 .{ }^{1,11}$ As such, Fig. 1 shows that trough configurations do not have sufficient concentration ratios for reaching temperatures required to heat lunar regolith to temperatures required for oxygen production or similar processes.

\section{E. Fresnel Reflector}

In order to achieve high temperatures for ISRU thermal processes while minimizing re-radiation losses, high concentration ratio solar concentrators are needed. To achieve this reflector configuration in a composite without the need for complex curvature surfaces, DR Technologies developed a point focus Fresnel reflector configuration under an SBIR funded by NASA/GRC. The approach uses an array of simple curvature parabolic strips, each with a small line focus, and each oriented to overlay the line focus into a central focal area, thus simulating a point focus concentrator, as shown in Fig. 9.

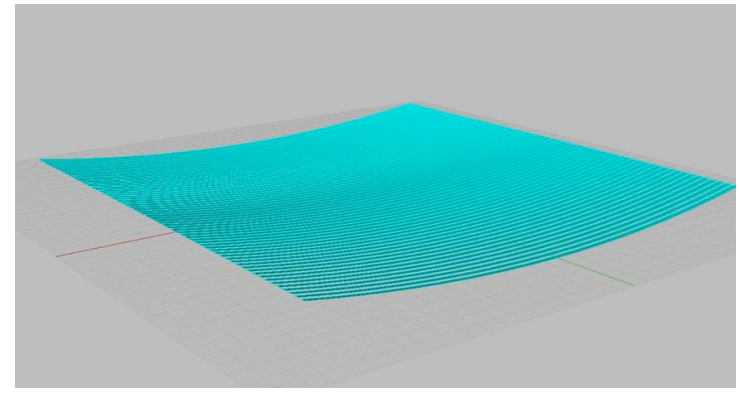

Figure 9a. Model 1.5 x 1.5 m Fresnel Reflector Using 60 Strips.

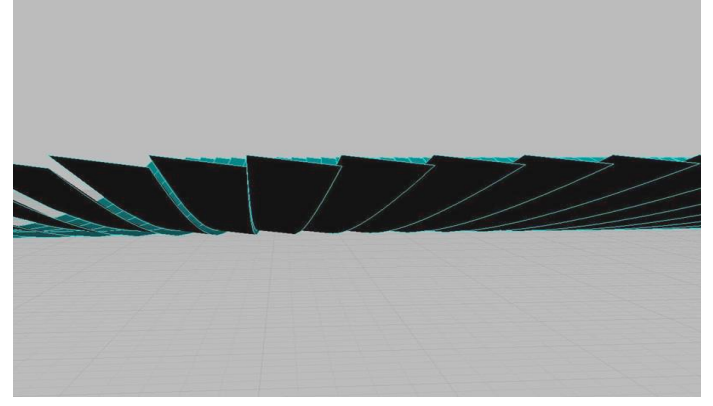

Figure 9b. Edge view showing individual strips.

This choice was based on several significant advantages. The array of simple curvature strips can be fabricated without using an accurately machined mandrel as would be needed for a parabaloidal dish, with each strip cut from a replicated mirror flat and then mounted on an accurately machined structure. The Fresnel reflector has a low 
cross-section, which makes packaging for shipment and launch considerably more efficient, and the independently mounted strips are less likely to incur global thermal distortion errors.

The concentrator design synthesis used the SOLTRACE code provided by the National Renewable Energy Laboratory. The model drove design trades that varied the width, length and number of Fresnel strips in the concentrator, the focal length, the overall aperture, and then determined the number and distribution of rays falling within a selected aperture size by ray tracing, while considering realistic mirror slope and specularity errors. These trades drove the design parameters to achieve high intercept efficiency with a geometrical concentration ratio of 2500, as shown in Figs. 10. The flux distribution is given in Fig. 11.

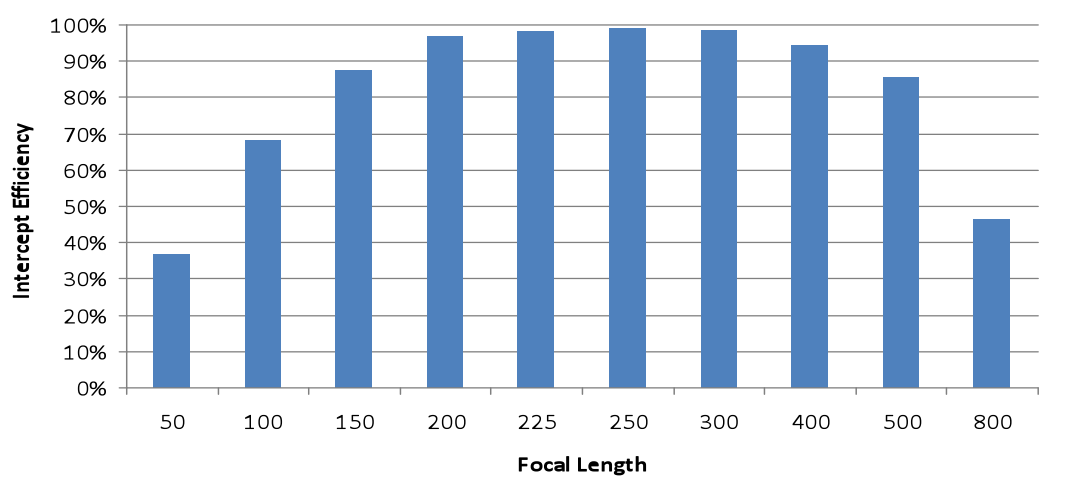

Figure 10. Intercept efficiency as a function of focal length for a $150 \mathrm{~cm}$ aperture.

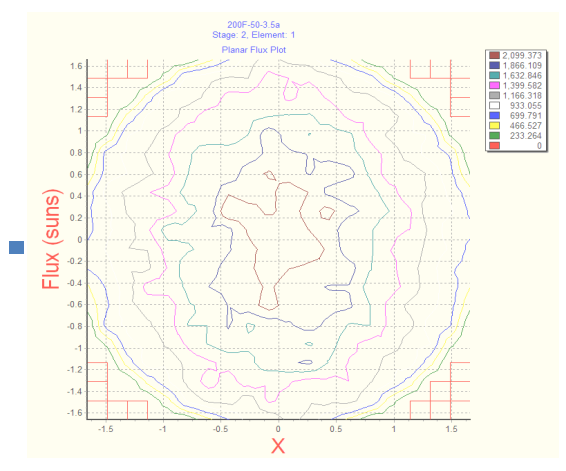

Figure 11. Flux distribution predicted by SOLTRACE for the optimized F/d of 1.5.
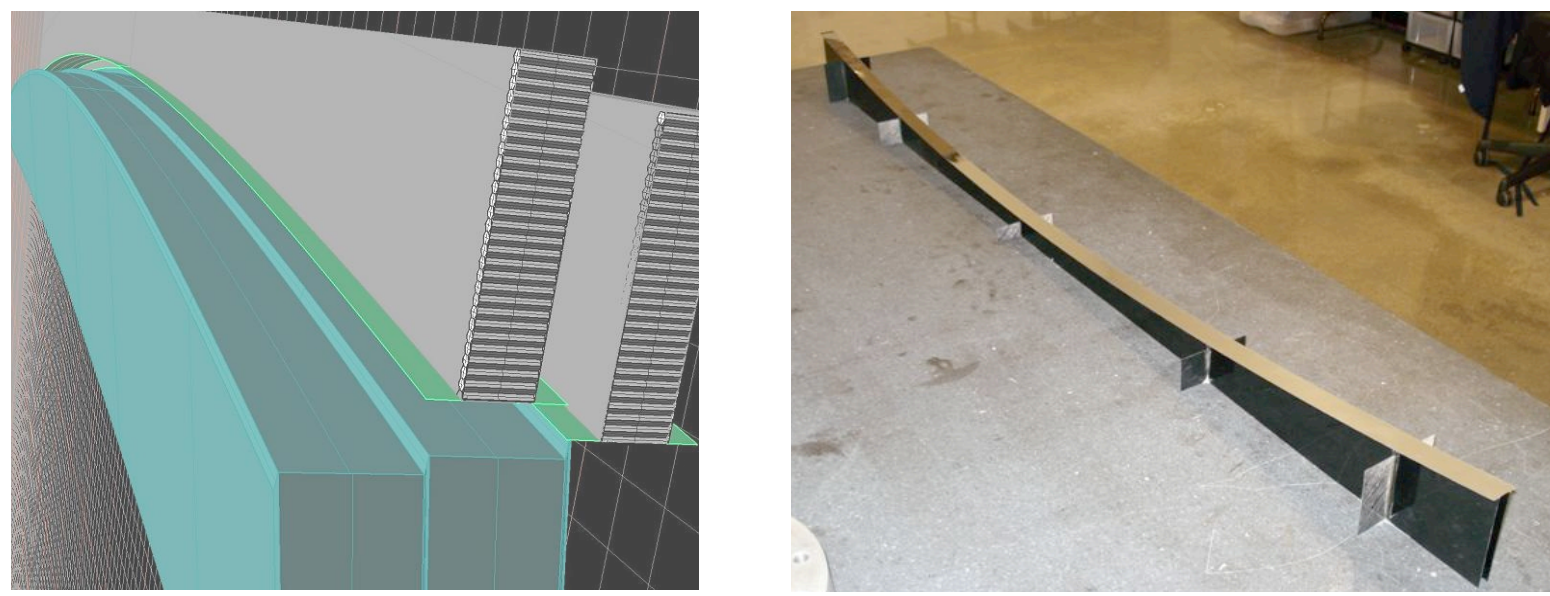

Figure 12. Implementation of Fresnel mirror on composite rib, and assembled on a positioning tool using a frame that fixed the mirror strip position.

A model of the Fresnel reflector was developed using mirror strips of Graphite Fiber Reinforced Composite (GFRC) fabricated using a proprietary mirror replication process for high specularity. Each mirror strip was mounted on a composite rib structure, as shown in Figs. 12 and 13, and assembled into a frame using a placement tool. Illumination tests on the concentrator using a heliostat showed the ability to achieve temperatures in excess of 1200C. Higher temperatures should be achievable with an optimized absorber and secondary optic configuration. 

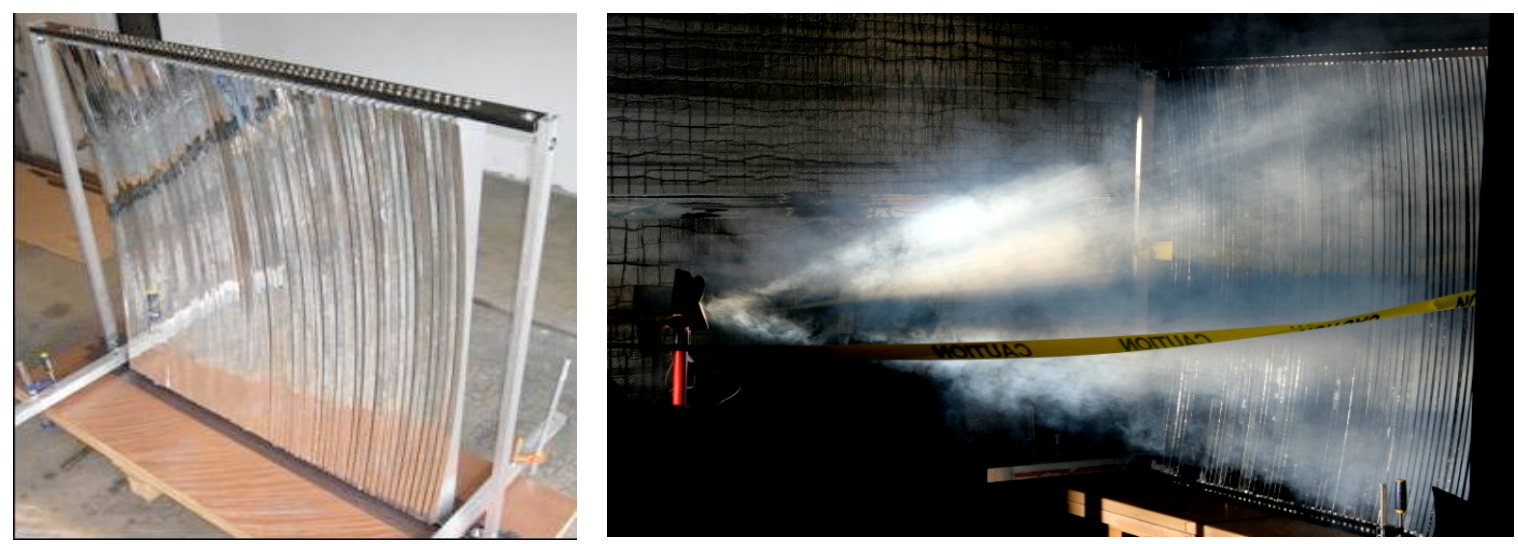

Figure 13. Mirror test article and illumination testing using fog machine for visual feedback.

\section{F. Secondary Concentrators}

Secondary concentrators are optical devices used to further concentrate focused light after being directed by an initial concentrator, or to move focused light to a desired location. Such technology has been designed and tested for effectiveness in the form of a sapphire refractive concentrator, shown in Fig. 14, with promising results. ${ }^{17}$ Although most secondary concentrators developed are lenses, nothing hinders a mirror from potentially being used as a secondary concentrator. The secondary concentrator could capture solar energy obtained from a potentially large concentrator, possibly of the flexible or inflatable varieties known for having lower CRs, into a smaller spot. Because the light is affected by another optical medium, there are inherent losses; the tested sapphire concentrator has a calculated transmission of $87 \% .{ }^{17}$ Losses could be minimized and the CR increased, however, with effective design and testing. The CPC's most effective task could be to act as a secondary concentrator to a Cassegrain or offset parabola. The secondary could also be used to direct solar flux into a designated position for prolonged time periods; the primary moving concentrator's spot could be pointed towards the secondary concentrator, which points towards the regolith or energy transfer device. Such a technology, however, would require complex control systems; such technology is likely to increase losses induced by sun tracking and inhibits its ability to capture end angle light rays. An example of a secondary lens system for focusing and further concentrating sunlight is shown in Fig. 15. This system utilizes a combination of lens and mirrors to be able

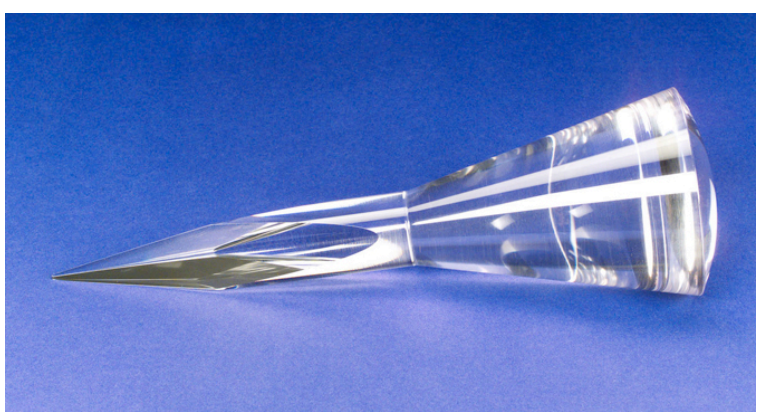

Figure 14. Refractive Secondary Concentrator.

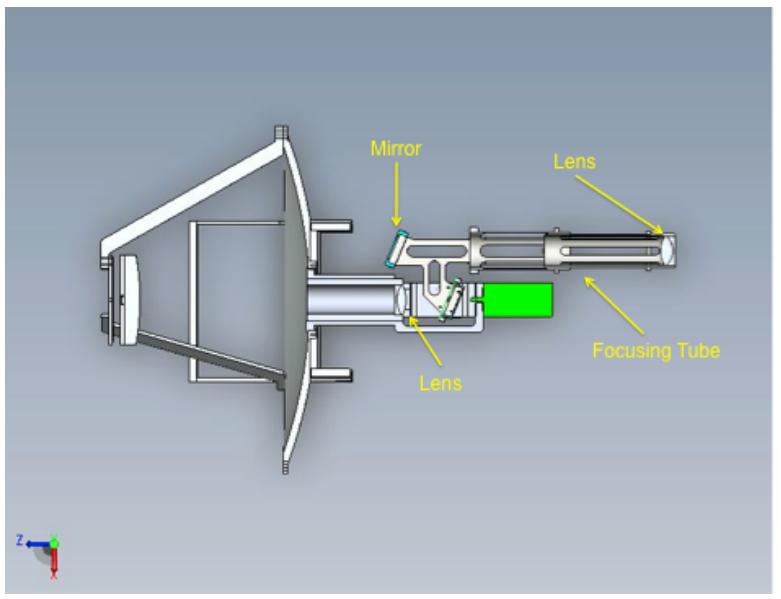

Figure 15. Cassegrainian Concentrator with Optical Concentrating \& Focusing System to concentrate and move the focus of the sunlight.

One of the largest issues with secondary concentrators, however, is likely to be the choice of material. Though sapphire was chosen for its ability to be used under extreme temperatures, it cracked and was rendered unusable once it reached $1550 \mathrm{~K} .{ }^{17}$ The primary concentrator chosen will focus solar light at least hundreds, preferably thousands, of times stronger than incident sunlight, and as such, the secondary concentrator must be that much more resistant to electromagnetic radiation and intense temperatures for prolonged periods of time. Slight 
imperfections in the secondary concentrator are likely to produce high operating temperatures due to the high light flux imparted to it from the primary concentrator. As such, the secondary concentrator should not be considered an afterthought, but an integral addition, to effective concentrator design.

\section{Reactor Designs}

Producing oxygen in-situ on the moon can provide significant mission benefits and overall cost savings to future missions. Earth is a relatively large gravity well that has an escape velocity of $10.4 \mathrm{~km} / \mathrm{s} .{ }^{5}$ This escape velocity requires tremendous resources to launch any significant object into orbit, and even more to bring along the fuel necessary to move once in orbit. The moon on the other hand has one-sixth the gravity of Earth. A launch from the moon to earth orbit would require much less propellant than launching a similar vehicle from Earth. The ability to effectively and efficiently produce oxygen from the material on the lunar surface must be realized to effectively harness these benefits. The carbothermal and hydrogen reduction processes for producing oxygen from the lunar regolith have the potential to achieve that goal.

\section{A. Hydrogen Reduction}

Hydrogen reduction makes use of ilmenite $\left(\mathrm{FeTiO}_{3}\right)$ in the lunar regolith as well as provided $\mathrm{H}_{2}$ gas to produce pure iron (Fe), $\mathrm{TiO}_{2}$ and $\mathrm{H}_{2} \mathrm{O}$. The temperature required to carry out this reaction is $1273 \mathrm{~K}$. A current design, shown in Fig. 16, of a hydrogen reduction reactor utilizes an auger to move the regolith through the reactor as well as heating the regolith with the blades. ${ }^{18,19}$ A heat pipe would be used to evenly heat the auger by distributing the energy from the solar concentrator which focuses light onto the heat pipe. The blades of the auger could also be used to introduce hydrogen into the regolith providing a fluidized bed of regolith. There is great potential for this process, but there still remains a number of challenges in the design and operation of the reactor. For example a process called hydrogen embrittlement caused by hydrogen gas permeating a metal structure can cause certain metals to crack, promoting premature failure. Some metals and alloys are more susceptible to hydrogen embrittlement than others.

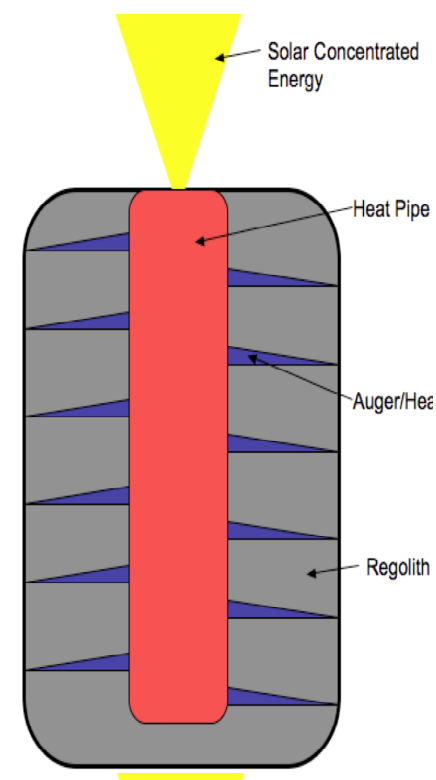

Figure 16. Simplified diagram of current hydrogen reduction reactor design.

\section{B. Carbothermal Reduction}

The carbothermal reduction method uses a temperature of at least $1800 \mathrm{~K}$ to extract the oxygen from all the major constituents of the lunar regolith as shown in Table 1 above. The current system being designed by Orbital Technologies Corporation (ORBITEC) is detailed in Fig. 17. ${ }^{20}$ The carbothermal reactor must melt the regolith to start the reaction; this melt is achieved by heating a small amount of regolith and using the surrounding regolith as insulation. Concentrated solar energy is used to melt the regolith. Excess space above the regolith is used to flow the reacting gas, methane. The gas reacts with the molten regolith to produce water, which is then electrolyzed to produce oxygen and hydrogen. The hydrogen gas is reused in the process to regenerate the methane.

The solar energy is focused through a window into the reactor chamber. There are other methods that could be implemented to getting the concentrated solar energy onto the regolith, including heat pipes or fiber optic lines. There are some potential design concerns with each of these approaches. For example by using a window to let concentrated sunlight into the chamber the window could be obscured by regolith particles in the reactor clinging to the window. Also the chamber has free flowing gas, which could pickup dust and interfere with the focusing of the light. It should be noted that a sufficient gap is required between the regolith and the window otherwise, the temperature gradient across the window can cause excessive thermal stresses potentially causing failure. 


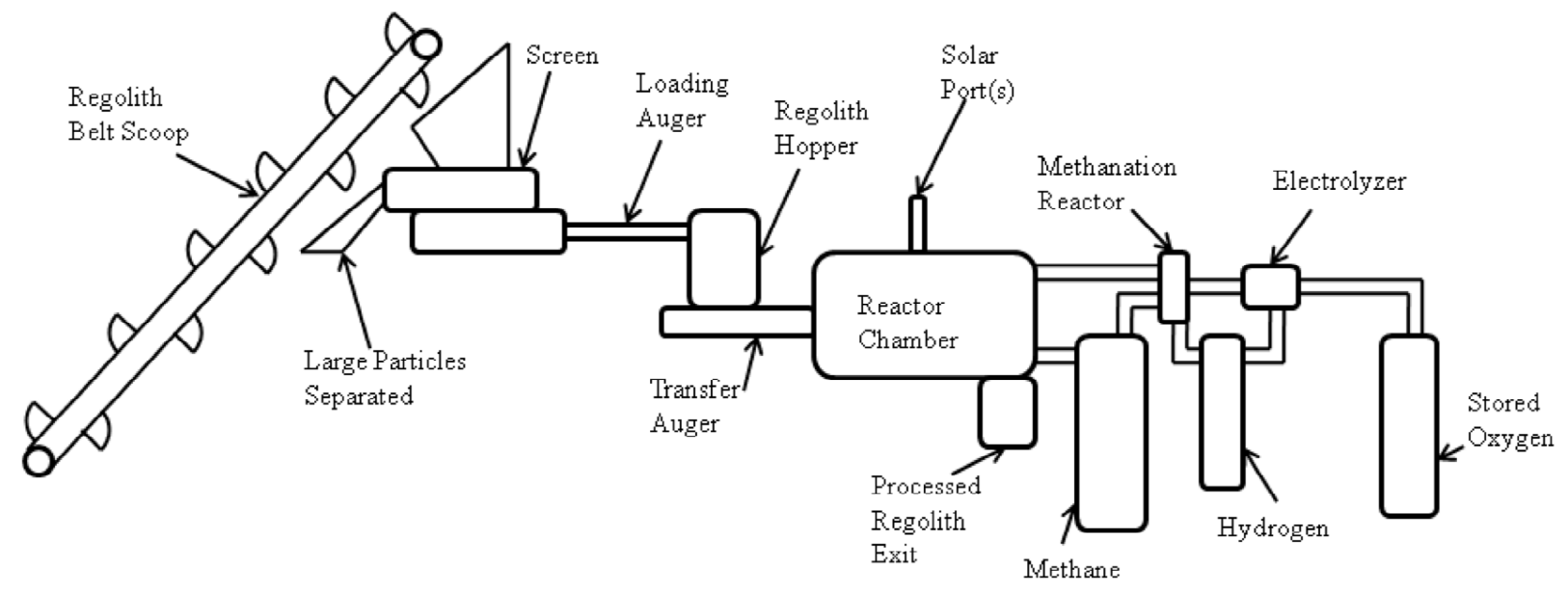

Figure 17. Diagram of current Orbitec carbothermal reduction reactor with features identified.

The ORBITEC Carbothermal Regolith Reduction Module was initially tested with a $\mathrm{CO}_{2}$ laser to simulate concentrated solar energy. The $\mathrm{CO}_{2}$ laser energy passed through a zinc selenide window and illuminated a bed of lunar regolith simulant located at the bottom of the sealed processing chamber within the Carbothermal Regolith Reduction Module. The laser energy absorbed by the regolith simulant caused rapid, localized heating. If laser energy flux was high enough, a pool of molten simulant would form surrounded by unmelted simulant due to the low thermal conductivity of regolith simulant. The surface temperature of molten simulant was determined by the laser energy flux. This heating approach worked well as long as the zinc selenide laser window remained completely clean during operation. Surface temperatures of the molten regolith simulant in excess of $1800^{\circ} \mathrm{C}$ were easy to achieve and maintain.

If any deposits or particulates accumulated on the window, some of the laser energy would be absorbed causing window heating and eventual failure. A solar concentrator system was later developed by Physical

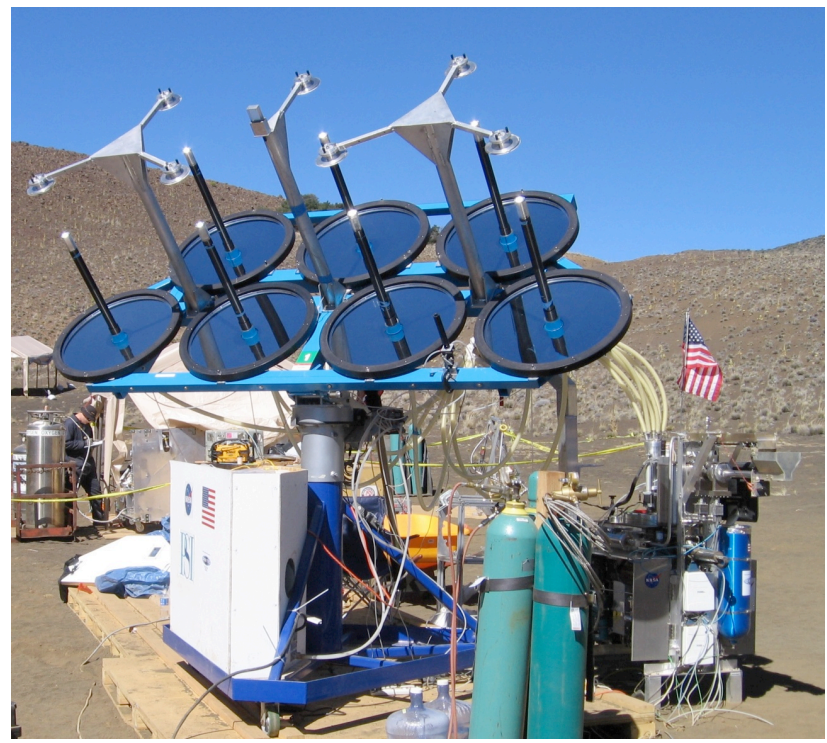

Figure 18. Photograph of the Solar Concentrator Array (left) with the Carbothermal Regolith Reduction Module hardware (right). Sciences Inc. (PSI) and integrated with the Carbothermal Regolith Reduction Module. The integrated system was successfully operated for nearly two weeks during the 2010 International Lunar Surface Operations and ISRU Analog Test on Mauna Kea in Hawaii shown in Fig. 18. Concentrated solar energy was delivered into the processing chamber through a quartz rod, as shown in Fig. 19. Since the temperature of the regolith surface was determined by the flux of the solar energy, varying the distance of the quartz rod above the regolith controlled the regolith temperature.

Similar to the zinc selenide laser window, the quartz rod efficiently transmitted the concentrated solar energy into the processing chamber when it was clean. However, transmission performance of the quartz rod degrades rapidly if any deposits or particulates accumulate on its surface. Deposits on the end of the quartz rod will absorb some of the concentrated solar energy, both attenuating the solar energy delivered to the regolith and quickly heating the quartz rod until failure. Unfortunately, keeping the quartz rod clean within the processing chamber proved to be more difficult than expected. 
The end of the quartz rod needed to be located 2.5-4 $\mathrm{cm}$ above the surface of the regolith simulant to maintain the temperature of the molten regolith at $1700-1800^{\circ} \mathrm{C}$. The carbothermal reduction of regolith produces silica vapor that will coat the interior surfaces of the processing chamber. In addition, rapid production of carbon monoxide gas in the molten regolith causes small bits of molten regolith to splatter on nearby surfaces as the gas bubbles break. To prevent potential silica vapor deposits or molten simulant splatter from accumulating on the end of the quartz rod, ORBITEC enclosed the end of the quartz rod with a clear quartz window. A thin layer gas blowing across the bottom of the quartz window was used to keep the quartz window clean during processing. Fig. 20 shows the interior of the processing chamber after a carbothermal reduction-processing batch. Note the layer of silica vapor that extends from the processed regolith simulant. When the proper gas flow rate is used, the silica vapor was blown away from the processed regolith

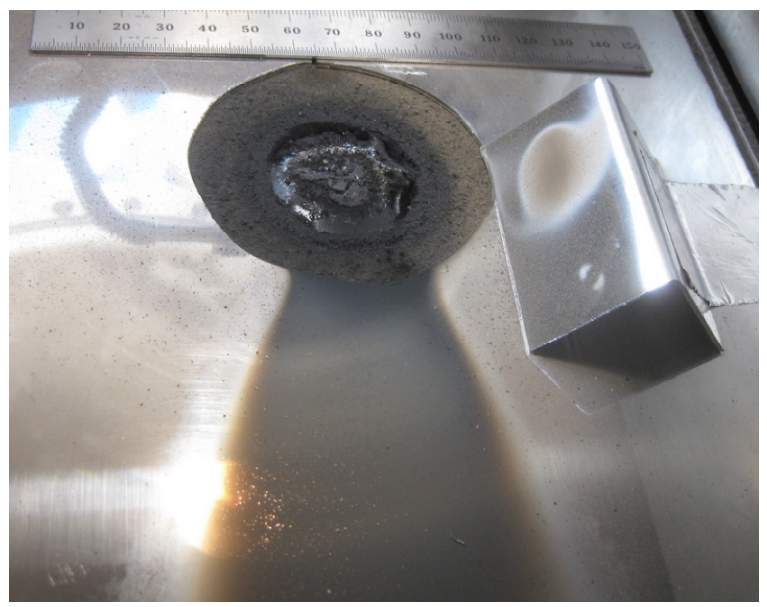

Figure 20. Example of silica vapor produced during carbothermal reduction processing of JSC-1A lunar regolith simulant with solar energy.

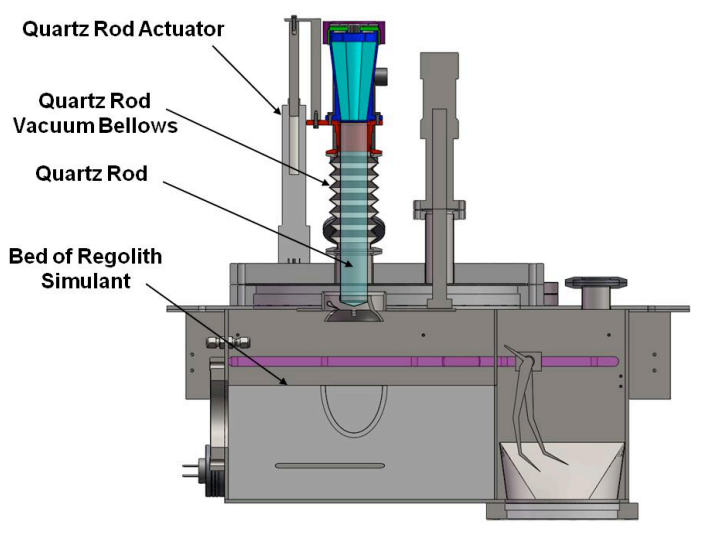

Figure 19. Cross-section View of the Processing Chamber within the Carbothermal Regolith Reduction Module.

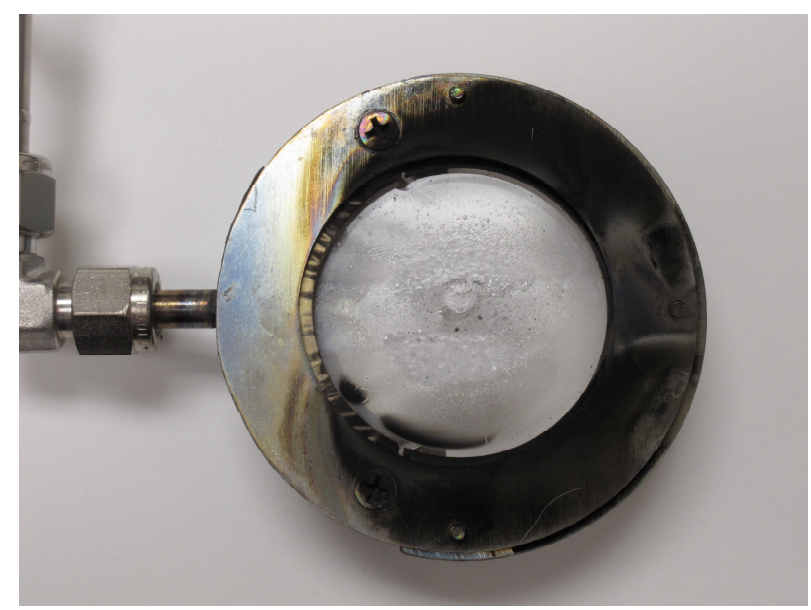

Figure 21. Damge to a protective quartz window due to accumulated silica vapor.

by the gas flowing across the bottom of the quartz window. If the gas flow is not correct, silica vapor can still accumulate on the quartz window and damage it as seen in Fig. 21. Although the layer of gas blowing across the quartz window was very effective at preventing any silica vapor from accumulating on the window, glass splatter from the molten regolith remained a problem if the bottom of the quartz rod was moved less than $3 \mathrm{~cm}$ above the regolith surface during processing. Fig. 22 shows a photograph of the quartz window after a carbothermal reduction-processing batch where the end of quartz rod was located $2.5 \mathrm{~cm}$ above the surface of the regolith. Note the numerous glass beads on the quartz window surface.

ORBITEC measured the reduction in the loss in the delivered solar energy due to the protective quartz window. Quartz windows with and without anti-reflection coatings were tested. The energy loss with an uncoated quartz window was $\sim 7.5 \%$ due to Fresnel reflection loss. When a quartz window with a VIS-IR anti-reflection coating was used, the energy loss was reduced to less than $1 \%$. However, when a coated quartz window was used for a carbothermal reduction test, the anti-reflection coating was damaged and began to flake off, as seen in Fig. 22. It appears that the window became too hot and the anti-reflection failed. An uncoated quartz window was used in subsequent tests of the Carbothermal Regolith Reduction Module. 

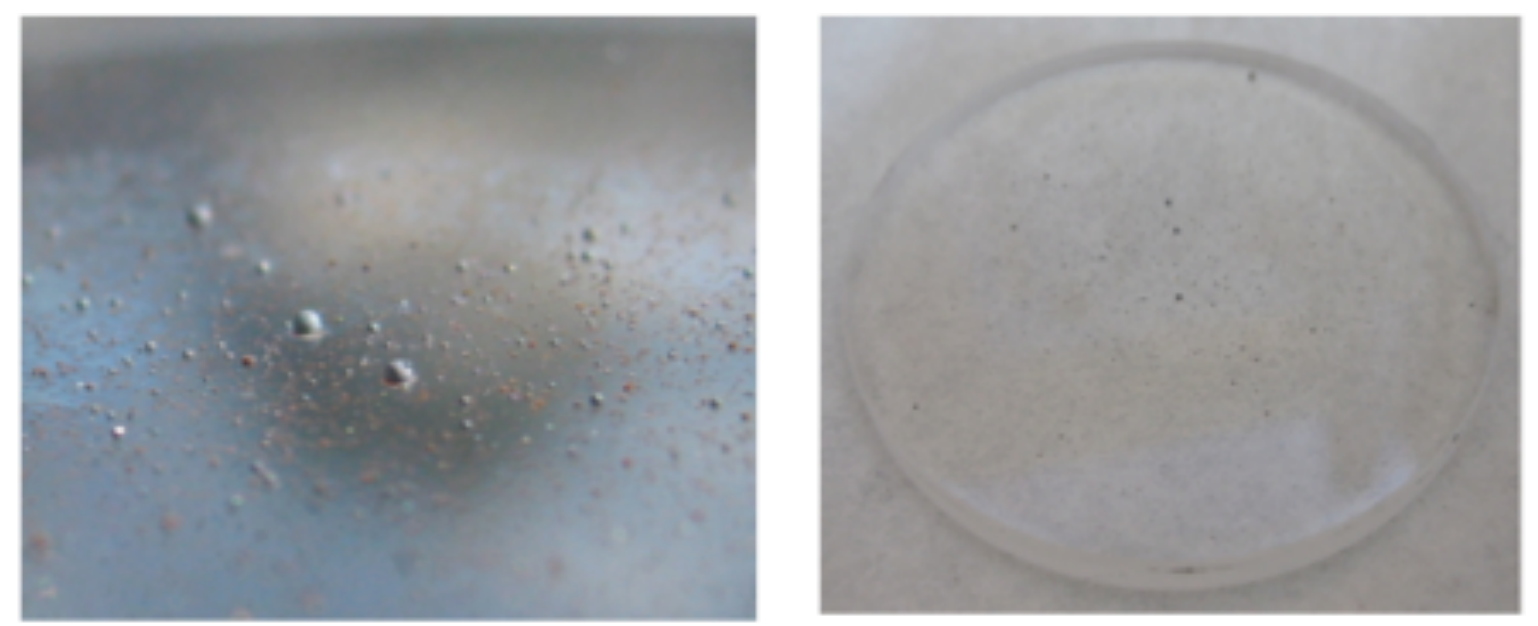

Figure 22. Small glass beads accumulated on bottom of protective quartz window (left) and magnified view (right).

There are several important lessons learned from this project. First, a quartz rod is an effective and efficient method to deliver highly concentrated solar energy into a carbothermal reduction-processing chamber. Using this delivery approach, molten regolith surface temperatures in excess of $1800^{\circ} \mathrm{C}$ were easily achieved. Second, varying the distance between the end of the quartz rod and the regolith surface can control the temperature of the molten regolith. Third, special precautions must be made to protect any optical surfaces within a carbothermal reductionprocessing chamber, such as the end of the quartz rod. Any deposits or accumulation of particulates will lead to rapid heat and degradation of optical surfaces. Fourth, anti-reflection coatings can be very effective at reducing Fresnel reflection losses. However, care must be taken to ensure that the coating can survive the thermal environment.

\section{Design Implementation}

In considering the complete system for material processing on the lunar surface, there are many more design considerations that need to be taken into account in addition to the concentrator geometry. To operate within the lunar surface environment, the concentrator must survive launch and be resistant to the lunar landscape. The presence of no appreciable atmosphere, solar wind, micrometeorites, electromagnetic radiation, and constant thermal cycling of the range of $\sim 300 \mathrm{~K}$ are primary issues to consider. The available technologies, such as lenses vs. mirror concentrators, and rigid vs. flexible concentrators, must be compared and contrasted to determine the applicability of each technology. The solar energy transfer options available to harness and transfer concentrator energy to heat the regolith must be addressed as well. Though this is not an exhaustive list of every necessary design consideration, it does provide an overview of design issues that must be considered when developing effective lunar-based solar concentrators.

\section{A. Mirror vs. Lens Concentrators}

Typically mirror-based concentrators are usually developed for thermal applications and lens-based concentrators have been associated with PV applications, however, definitive reasons as to why these correlations have been made are based largely on history and tradition. ${ }^{1}$ The environment, design, manufacturing, and operation of these technologies as lunar concentrators are inherently complex. Design heritage, cost, launch vehicle capabilities, packaging, mass environmental compatibility and lifetime factor in to which concentrating technology works in what application. In addition, because mirror concentrator designs for thermal applications are well researched, tested, and developed, mirror concentrators are likely developed and studied for thermal applications. The same goes for lens concentrators made for PV applications. 
From a physics standpoint, lenses are actually a much better choice for concentrator material. The maximum concentration ratio of a solar image on the absorber of a three-dimensional mirror concentrator, bounded by the thermodynamic limit for solar concentration, is described by Eq. $4:^{1}$

Theoretical optical constraints dictate that a solar concentrator made from a refractive material can reach higher concentrations than reflective concentrators, as defined by the equation $8 .{ }^{1}$

$$
C R_{3 D \max , T h}=\left(\frac{n}{n_{\text {surr }} \sin \left(\theta_{s}\right)}\right)^{2} \approx 97,700
$$

The index of refraction $n_{\text {surr }}$ in air equals 1 and $n$ for crown glass equals $1.5 .{ }^{1}$ However, lens effects such as spectral shifting and groove darkening reduce the actual light concentration capability of a lens. Because of intrinsic physical deficiencies of lenses such as these, in real world applications mirror reflectors usually have a higher CR than most lens concentrators. However, lens do have some advantages over mirrors: angle incidence errors caused by errors in sun tracking or concentrator shape distortions are drastically less with lenses than with mirrors. Fresnel lenses with the unique arch shape of the Stretched Lens Array actually provide more than two orders of magnitude better shape error tolerance than reflective concentrators, or than conventional flat Fresnel lens concentrators; when the material does get damaged, it's more likely to continue concentrating light effectively. ${ }^{2}$ In fact, for assumed mirror-based thermal concentrators, the accuracy of the tracking mechanism needs to be approximately one hundred times better than that for lens-based concentrators. ${ }^{1}$

The issues with utilizing lenses occur when considering manufacturing techniques and possible materials for lunar use. Lens materials usually come in either glass, or transparent plastics. When glass lenses are used as primary lens material, concentrator become very heavy. Previous ISRU oxygen production studies calculate the concentrator or concentrator assembly needs to capture approximately $14.38 \mathrm{~m}^{2}$ of solar flux at minimum, which would need large, heavy lenses as primary concentrators. ${ }^{3}$ What's more, glass is a brittle material that has the potential to crack or shatter due to intense stresses experienced by launch. Plastics, such as space-qualified silicone DC 93-500 used for the Stretched-Lens Array, must be held in place through external structure. ${ }^{2}$ Plastics are also easily affected by the space environment effects such as atomic oxygen, UV, micrometeorites and must be protected by films from degrading. The metals and composites used in mirror materials are much less likely to be affected by environmental effects. Scaling of the technologies is also a factor; it is traditionally easier and lower cost to make larger mirrors with high CRs than larger lens concentrators with the same CRs.

Because tradition has linked mirror concentrators and thermal applications, and lens concentrators and PV applications, companies specializing in the manufacture of such technologies have been formed which reinforce such traditional links. However, concentrator options are widely available for multiple functions by different companies: Optiforms, Optical Mechanics, and Hardric Laboratories specialize in the manufacturing of mirrors meant for a spot focus. Minnesota Mining and Manufacturing (3M) manufactures sheets of space-rated silicone for line-focus concentrators of any size, but there are minimal companies who specialize in the creation of point-focus Fresnel lens concentrators. ${ }^{2}$

\section{B. Rigid vs. Inflatable/Flexible Concentrators}

Rigid concentrators are those that are self-supporting; as in, the concentrator has enough structural rigidity to keep its shape without outside influence. Flexible concentrators (Fig. 23), however, forego structural rigidity for very low weight and small storage volume. ${ }^{10}$ They must, however, be held into shape by some external structure during operation or rigidize after being deployed. One approach is that of an inflatable concentrator kept rigid by trapped gas inside. Such technology has a much higher specific power rating, or power per unit mass, than rigid concentrators. ${ }^{18}$ This means inflatable concentrators can be made much larger than rigid concentrators of the same weight, and capture much more solar flux. Inflatable solar concentrators have been made capture an effective solar flux diameter of over $4 \mathrm{~m} .{ }^{18}$ Flexibles and inflatables, however, usually maintain their shape by a combination of some supporting back structure along with the inflated gas. Such technologies however, have a much higher shape error than a polished rigid mirror concentrator or a finely milled Fresnel lens concentrator.

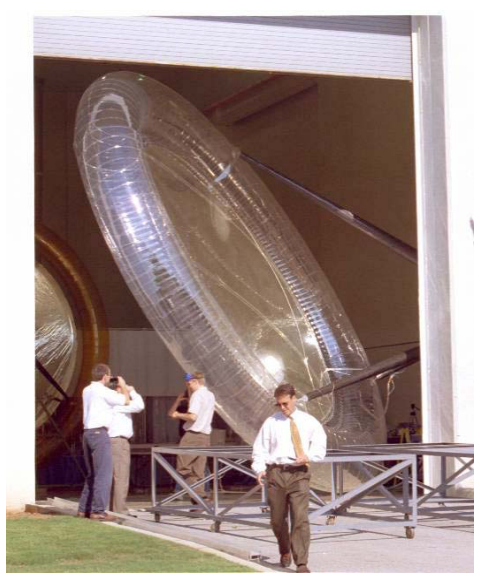

Figure 23. Flexible $4 \mathrm{~m}$ by $6 \mathrm{~m}$ offset parabolic concentrator. 
Thus, the spot size of inflatable concentrators is much larger than similarly sized fixed concentrators. Low-weight rigid concentrator assemblies are possible, like the rigid concentrator hexagonal assembly made for the James Webb Space Telescope. ${ }^{12}$ Using such techniques however, are likely to drastically increase manufacturing costs.

Although inflatable concentrators have advantages of lower mass and stowage volume over rigid concentrators, there are other issues that limit their applicability. The inflated area that actually focuses light has to be inflated from both sides, which means for mirror concentrators, the first material the incident light must travel through must be transparent. Most materials that are not gas permeable and can take extended operation in a vacuum are not completely transmissible, and possible losses over the usable EM spectrum increase. Another important issue with flexible concentrators is the effective life of such technology in the hazardous space environment; most usable plastics are less resistant to space and lunar environmental effects than metals and glass. Micrometeorite puncture analyses have been performed for flexible structures in LEO, but lunar projectile fluxes have yet to be studied in detail. Atomic Oxygen causes a chemical reaction with space inflatables that can erode or build up a growth on the polymer surface, causing degradation of the material. Ultraviolet radiation and solar wind serves to break chemical bonds and to decrease tensile modulus in exposed materials. Interestingly enough, silicone varieties are some of the most resistant materials to harmful radiation, and silicon oxides are particularly resistant to atomic oxygen effects. ${ }^{21}$ UV darkening is known to occur for many plastic materials, and as such, UV coatings to protect space-rated silicon are being developed with promising results, but such technologies must undergo sufficient proving ground testing. ${ }^{2}$ The transparency of specific materials with coatings and their wear over prolonged periods of time remains to be studied.

\section{Rigid Mirror Concentrator Material Overview}

The desired material requirements for a rigid mirror concentrator technology are as follows:

- High Reflectivity

- Low Coefficient of Thermal Expansion (CTE)

- Strong (High Yield Stress, High Modulus of Elasticity)

- $\quad$ Light (Low Density)

- Inexpensive, if possible

- Reliability over prolonged periods of operation during harsh lunar exposure

Most of the material requirements are more obvious than others. For example, a high yield stress and high modulus of elasticity means the material is strong and resistant to deformation. Low-density materials mean lighter weight structures and lower cost to launch. Reliability means the material will not break, creep, falter, or wear in the multitudinous ways in which material breakdown is feasible. A low CTE means that during the large temperature swings on the moon, the material is resistant to effects of thermal stresses and deformation.

Most materials considered for mirrors do not have all of these qualities; silver, gold, and copper, which have the highest reflectivity, have high CTE's and are heavy. Aluminum, which can produce a mirror finish with a reflectivity of $91 \%$, is light and strong, but has a high CTE. ${ }^{13,22}$ As such, composite mirror technologies are being developed where a light and strong substrate is used for the structure and a reflective material is deposited on its smooth surface. Atomic layer deposition (ALD) processes are being considered for the mirror application process so the deposited film is thin enough such that there are minimal thermal stresses between the substrate and the mirror coating. Mirror deposition for optical thin films has been shown to be effective with deposited layers on the sub-micrometer level, but more research must be conducted on substrate-mirror composites for lunar-based solar concentration to demonstrate they can be made with sufficient surface quality and the desired reflective coatings can be applied. ${ }^{23}$

\begin{tabular}{|c|c|c|c|c|}
\hline Ele ment & Melting te mp (K) & CTF $\left(\mathbf{1 0}^{\mathbf{6}} / \mathbf{K}\right)$ & De nsity $\mathbf{~ k g} / \mathbf{m}^{3}$ & Bulk Modulus (GPa) \\
\hline $\mathrm{B}$ & 2349 & 6 & 2460 & 320 \\
$\mathrm{C}$ & 3800 & 7.1 & 2267 & 33 \\
$\mathrm{Mg}$ & 923 & 8.2 & 1738 & 45 \\
$\mathrm{Si}$ & 1687 & 2.6 & 2330 & 100 \\
$\mathrm{Ti}$ & 1941 & 8.6 & 4507 & 110 \\
\hline
\end{tabular}

Figure 24. Graphical comparison of candidate elemental substrate materials chosen for optimal values of melting temperature, coefficient of thermal expansion (CTE), density, and bulk modulus of elasticity. 
A preliminary study of possible elements that could be utilized as mirror substrates is necessary, and as such, there are multiple elements that are potential choices. Figure 24 compares five candidate elements for mirror substrates and their physical properties, which will impact their effectiveness: CTE, density, melting temperature, and module of elasticity are shown. silicon, boron, carbon, magnesium, and titanium are all potential substrate candidates due to their high strength, their relatively low density and their low CTE. Notably, silicon has the lowest CTE, and titanium, known for its applications in aerospace technology, has the highest. A detailed analysis of using advanced materials such as ceramics, composites or metallic alloys as mirror substrates should also be considered for future research. ${ }^{24-29}$ The unique properties of these engineered materials would need to be evaluated to access their applicability to the lunar and space environment.

In addition some far-term technologies and considerations include the use of electroactive materials, known as Ionic Polymer Metal Composites (IPMC's) as mirror substrates. Originally conceived as an alternative for prosthetic muscle material, the mirror surface itself could deform to adjust to different conditions and a means of actively focusing the mirror. The polymer accomplishes this by deforming when exposed to an electric field. This type of technology holds significant promise in providing the ability to fine tune the focus of the mirror while it is operating as well as redirect the focus and potentially aid in tracking.

\section{Lunar Environment}

The lunar environment provides several design challenges as well as opportunities. The moon has a gravitational attraction equal to about $1 / 6$ that of Earth. The lower gravity can make it difficult for humans to walk in. However for structures and some processes, the lower gravity is beneficial. Solar concentrators on Earth require bulky and heavy support structures to keep the mirrors from distorting due to gravity and wind loading.

The moon has a very hard vacuum of $\sim 10^{-12}$ Torr. $^{30}$ There are several consequences of this high vacuum; primarily, there is no weather. The majority of deflections in parabolic systems on Earth are due to the forces exerted by the wind especially during a storm with heavy winds. On the moon, except for the solar wind, there are no appreciable wind forces to cause any additional deflections. Another advantage to the vacuum is heat transfer. Without an atmosphere, there is no convection or conduction process, save conduction through the lunar surface. This leaves radiation as the only appreciable heat exchange mechanism. This is advantageous for the heating and processing of lunar materials since heat losses can be minimized. A downside, however, is that the reaction must take place in a pressure vessel to maintain the internal gas pressure and provide a means of capturing the reactants for processing. With radiation as the only means of heat transfer; temperature gradients across devices and components can become extreme because there is no atmosphere to help even out the heat transfer.

One of the biggest environmental challenges on the moon is the lunar dust. The average particle size of the constituents of the lunar regolith is about $70 \mu \mathrm{m}$, with $20 \%$ by weight of the regolith being less than $20 \mu \mathrm{m} .{ }^{31}$ Because of the small size and shape of the regolith, there are 3 primary challenges to solar concentrator and oxygen production systems: dust clinging, abrasiveness, and clogging. ${ }^{30-32}$

i. Dust Clinging - The main concern with the dust clinging is the adhesion to the optical surfaces. The dust is electrically charged allowing it to stick to a variety of surfaces. ${ }^{31}$ A mirror lens, or window will transfer less light while coated in dust, which will greatly diminish the efficiency of the system. Some technique must be devised to either keep the dust off of the concentrator and other optical components or be able to clear away dust that will inevitably coat the optical surfaces during operation.

ii. Abrasiveness - Due to the abrasiveness of the dust it can also obscure and damage optical surfaces due to the abrasiveness of the dust. During Apollo 17, scratching on Harrison Schmitt's sun shade prevented him from seeing in some directions. ${ }^{30}$ Extensive scratching of solar concentrator mirrors would cause light to scatter due to the small changes in the surface, which would once again negatively affect the efficiency of the system.

iii. Clogging - Dust clinging can also clog pressure vessels and seals. This can affect seals as well as inlets and outlets, gears and motors. This issue will be very difficult with the oxygen reactors. Both the carbothermal and hydrogen reduction methods use gases for their reactions, which will create a pressure inside the reactor. With the vacuum of space outside the reactor, some pressure seal will need to be in place. However, the dust cannot be avoided because it is to be used in the reactor. Care will need to be taken for any moving part or opening, so that dust clogging doesn't occur.

The last potential environmental issue to be discussed is vacuum welding of moving parts. When two parts move relative to each other, they have friction and are often in close contact causing the surfaces of the two parts to scratch each other. On Earth there is an abundance of oxygen, which reacts with the exposed material to create a nonreactive coating. In a vacuum, there are no gasses to react with. This allows the materials of the moving 
system to bond together, and potentially seize up the mechanism. It will be a challenge to ensure that the tracking system on a lunar solar tracker or the moving parts in the reactor do not vacuum weld together.

\section{E. Geographical Location}

The selection of a specific concentrator technology will also be greatly influenced by lunar location; each place has its advantages and disadvantages. On the lunar surface there are two main types of soils available. These consist of the older, brighter soils at high topographies, termed highlands, and the darker basaltic lava flows of the mare located at lower topographies. ${ }^{3}$ A display of the topography on the moon is shown in Fig. $25 .{ }^{34}$ The regolith at the highlands and Mare have different compositions and therefore different oxygen production processes are applicable to each of these areas. The hydrogen reduction process for producing oxygen can yield $1 \%$ to $5 \%$ of oxygen per unit volume of ilmenite. ${ }^{33}$ For comparison the carbothermal oxygen production process requires high enough temperatures to melt the regolith $(\sim 1800 \mathrm{~K})$, but can be used with most of the metal oxide compounds found in the regolith. The oxygen yield of the process is approximately $15 \%$ by weight. ${ }^{3}$

For locations within the mare regions, either hydrogen reduction or carbothermal reduction can be used as an oxygen production process. Since ilmenite is not found in usable amounts in the highland regions, ${ }^{35}$ the carbothermal process would be utilized within these regions. This means higher temperatures and more precise concentrator optics are required.

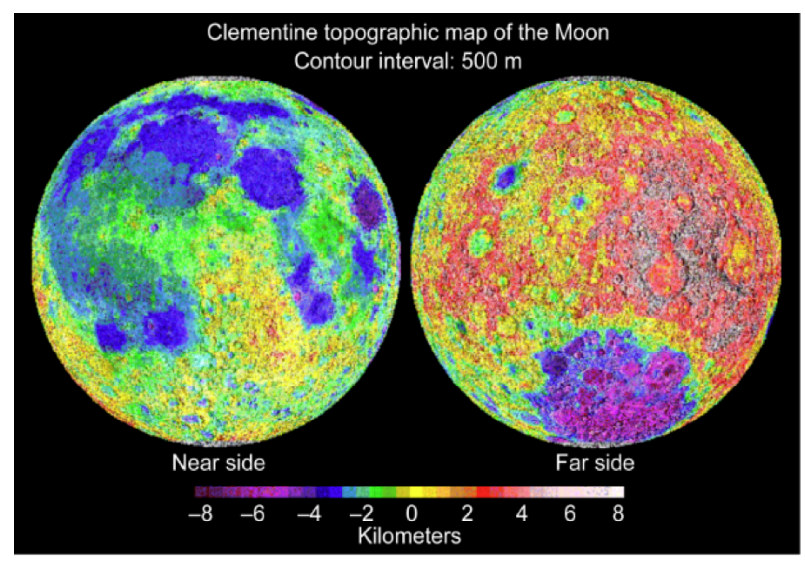

Figure 25. Topographic map of the Moon determined by the Clementine mission of 1999.

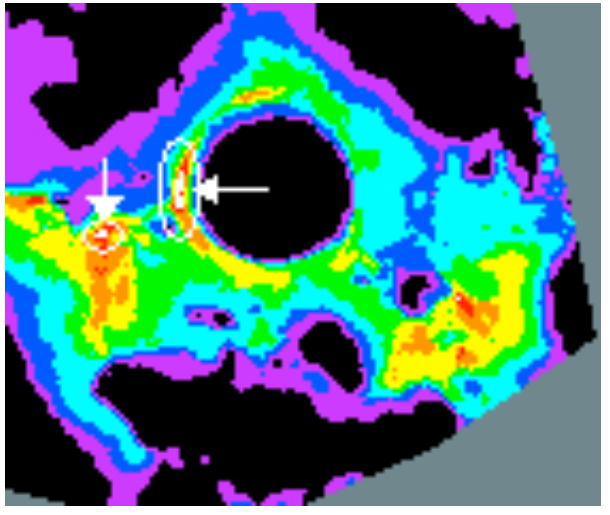

Figure 26. Percentage of time with solar illumination during lunar winter. The arrows are pointing at the two regions that are illuminated $>70 \%$ of the time during lunar winter; near the Shackleton Rim.

Though both the mare and the highlands have different regolith characteristics, they both have one significant problem; for approximately 14 Earth days, most of the mare and highland topographies are enshrouded in darkness. ${ }^{36}$ Because the Moon's axis is angled 1.5 degrees from the ecliptic, the South pole is almost constantly bathed in sunlight; during lunar summer, the entire South Pole is visible, and during lunar winter, the Shackleton crater and other small southern regions are illuminated by the Sun for more than $70 \%$ of the time. ${ }^{36}$ Figure 26 shows the percentage of time regions near the South Pole geographies are illuminated during lunar winter. Such areas would be an ideal place for a constantly operating solar concentrator that could generate approximately twice the amount of oxygen than an ISRU system anywhere else on the moon. However, there are minimal deposits of iron and titanium located at the poles. ${ }^{35}$ Less than $1 \%$ of ilmenite is present which means hydrogen reduction would not be the preferred oxygen production method; carbothermal reduction or other non-mineral-specific processes must be pursued.

Additionally, the long lunar days and nights along with no atmosphere result in large thermal cycling. The temperature range on the lunar surface is approximately $100 \mathrm{~K}$ during the night and $400 \mathrm{~K}$ during the day. One potential problem could be materials thermally expanding. The equation for simple linear thermal expansion is:

$$
\Delta \mathrm{L}=\alpha \mathrm{L}_{\mathrm{o}} \Delta \mathrm{T}
$$


Where $\Delta L$ is the change in the length, $\alpha$ is the thermal expansion coefficient of the material, $L_{o}$ is the starting length, and $\Delta T$ is the change in temperature. For a $1 \mathrm{~m}$ length of aluminum, the result shows that the length would grow $6.9 \mathrm{~mm}$ after the transition from night to day. Consideration would have to be taken to ensure that different parts of the system do not expand differing amounts and cause stresses, breakages, or unwanted gaps.

\section{F. Energy transfer options}

Most concentrators are required to track the sun to be effective, and as the sun moves, so does the concentrated spot of solar energy needed to heat regolith. Models of regolith heating cycles require that the regolith be heated by stationary spots for 2.5 hours before the regolith has been effectively processed.$^{37}$ Reactor assemblies being used for research and production are too large to move with the progressing spot, so in most cases, the energy needs to be transferred to the stationary reactor. As such, there are multiple ideas for energy transport.

Direct illumination of the regolith within the reactor is one option that can be utilized under certain operational conditions. In such a configuration, the spot focused from the concentrator would strike the regolith directly. For example, the South Pole offset parabola configuration that tracks the sun's movement by rotating would ideally have a stationary spot, making direct illumination possible for hours at a time. ${ }^{7}$ Direct illumination would have the smallest energy losses since inefficiencies due to additional optical components used to direct and channel the concentrated sunlight would not be needed. Other than the efficiency of the concentrator mirror itself the only other source of loss would be the window in which the concentrated sunlight would pass to enter the reactor chamber. For other operating locations than the North and South poles and concentrator configurations additional optical components would be required to keep the spot in one place for extended periods of time. A secondary concentrator possibly made from a $\mathrm{CPC}$ or from a refractive concentrator lens might be a plausible alternative. ${ }^{17}$

Because of the low thermal conductivity of the regolith, the direct heating of the surface does not provide a good means of uniformly heating a batch of the Regolith. Only the top layer of soil would be effectively heated by direct illumination. For certain reactor designs this may be acceptable. For example, in a carbothermal reactor the reaction takes place at the regolith surface and the regolith below the surface is used to insulate the chamber from the high heat of the process. In other designs such as those for hydrogen reduction, uniform heating is more desirable and surface heating would not be the best approach.

Another form of energy transport which can be considered is the optical cable, as outlined in the solar concentrator system for ISRU oxygen production assembled by PSI. ${ }^{13} \mathrm{~A}$ secondary concentrator located at the primary concentrator's focus would take concentrated sunlight and guide it to a fiber optic cable bundle Which in turn would transfer and focus it onto a stationary position within the reactor. The flexibility of the fibers makes it possible for the concentrator to be tracking while illuminating a fixed spot within the reactor. Great strides towards effective optical cable technology have been made through PSI's efforts as shown in Fig. 27. There are inherent losses in this type of light transfer system due to the inefficiencies of the optical cable. The complete concentrator configuration assembled by PSI had an efficiency of $37 \%$, though use of higher quality components would increase system efficiency. ${ }^{13}$

Heat pipes are also being considered as an alternative technology for heat transfer, Fig. $28 .{ }^{37}$ In such a process, incident solar radiation would be focused onto a heat pipe. By using solar energy to evaporate and condense the working fluid within the heat pipe, the heat pipe would transfer heat to the regolith. Heat pipes provide isothermal heat transfer into the reactor, which increases the lunar regolith processing efficiency. ${ }^{37}$

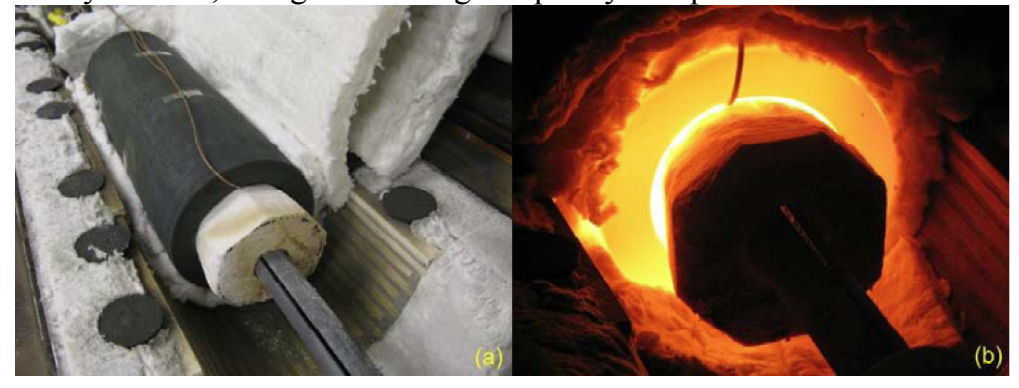

Figure 28. (a) Demonstration heat pipe test set up and (b) demonstration heat pipe operating at $1050^{\circ} \mathrm{C}$ using a Kanthal heating element. 
Heat pipes also make it possible to transfer heat throughout the reactor thereby providing more uniform heating than that achieved by direct surface illumination. Unlike an optical component, solar energy can be incident upon a heat pipe at very large acceptance angles, up to $90^{\circ}$. In contrast the fiber optic cables require the solar energy to be incident at an angle less than $20^{\circ}$. This enables the heat pipe to be capable of accepting incoming sunlight from the concentrator over a range of angles simplifying the tracking and transmission process of the concentrator.

There are concerns with the use of heat pipes for certain processes, particularly the carbothermal process. For the heat pipe to effectively transfer heat to the regolith it must be in direct contact. In the carbothermal process the high reaction temperatures and molten regolith provide a harsh and corrosive environment for the heat pipe. Material buildup on the heat pipe is a significant concern as well as corrosion and oxidization of the surface. This would lower the thermal conductivity of the heat pipe, and thus the effectiveness as well as potentially lead to its failure. Heat pipes are better suited for processes that remain below the melting temperature of the regolith, such as the hydrogen reduction process.

Heat pipes are rigid; designs either have the heat pipe attached to a tracking concentrator, or the heat pipe inside the reactor as a heat collection cavity. As such, most of the problems with keeping the spot stationary that apply to direct illumination also apply to heat piping technology. Mobile or flexible heat pipes might be an option for such a technology, however, and should be studied as well. More research needs to be conducted that verifies effective operation of heat pipes at temperatures in excess of $1625^{\circ} \mathrm{C}$, temperatures necessary for carbothermal reduction. ${ }^{20}$

\section{Conclusions}

Solar concentrator technology for ISRU-based oxygen production is an interdisciplinary topic that draws from many different technological achievements for inspiration. To reach the high temperatures necessary for regolith processing, different designs, materials, and energy transfer options have all been discussed, each with their inherent advantages and disadvantages. Each technology is useful for something, and with few exceptions, this paper does not boast the superiority or inferiority of a single design. The paper can be used, however, to compare and contrast the technologies available today for individual engineering purposes. Some technologies are adaptable for multiple oxygen reduction sites and can be used with multiple oxygen production processes, while other designs are much more specific. A Cassegrain concentrator system with an optical cable assembly, for example, could be placed anywhere on the moon, while an offset parabolic concentrator using direct illumination can be designed specifically for operation on the South Pole. ${ }^{7,13}$ It appears that the more modular the concentrator design, the lower the efficiency of the system; though such a tradeoff is expected, it is completely up to the designers of the system as to what design options should be pursued.

Though significant progress has been achieved for ISRU applications in a relatively short time, solar concentrator technology still must undergo a significant development effort before the technology should be put into operation. More research must be conducted on concentrator material design, specifically mirror substrates. Composites with a low CTE, high strength, high resistance to the harsh lunar environment, and strong bonding to reflective materials like silver, copper, and gold are required. Effects of stresses caused by thermal cycling, and the use of materials made of compounds, such as synthetics and metallic alloys, are all topics to consider. In the near future, more research needs to be conducted on the effect of lunar dust on concentrators. Plasma sprayers, compressed air, or simple window wipers are all being considered as dust mitigation alternatives for large mirror based concentrators. It has also been observed that that some of the lunar dust might bond to specific lunar regolith based upon deposition techniques. Some techniques might be more resistant than others, and such techniques must be effectively tested and optimized. Integration of multiple technologies and their combined effectiveness must also be investigated. It is entirely feasible to have a primary Cassegrain, a secondary concentrator, and a heat pipe working together to heat regolith; the efficiencies and inherent drawbacks of each system must be developed and studied in detail. Each specific technical challenge will require a unique technology solution.

\section{Acknowledgments}

We acknowledge support of this effort by the ISRU project of the NASA Exploration Technology Development and Demonstration program. We thank Dr. Kurt Sacksteder, Charlie Castle, Bob Macosko, Mike Piszczor, and Diane Linne at NASA GRC and Dr. Mark O'Neill of Entech Solar, Eric Cummings of Cool Earth Solar Inc., and Harvey J. Gibson, Jr. of Edmund Optics for technical discussions. Support of Pierce Gordon by the NASA Science and Technology Institute (NSTI) program supported by the UNCF Special Programs Corporation is acknowledged. Richard Heller is grateful to NASA GRC for support from the Graduate Student Researcher Program (GSRP). 


\section{References}

${ }^{1}$ Leutz, R., and Suzuki, A., Nonimaging Fresnel Lenses: Design And Performance Of Solar Concentrators, Springer-Verlag, Berlin, 2001, pp. 53-57.

${ }^{2}$ Piszczor, M.F., Jr., O'Neill, M.J., Eskenazi, M., and Brandhorst, H., "Stretched-Lens Photovoltaic Concentrator Arrays For NASA's Moon/Mars Exploration Missions," $2^{\text {nd }}$ International Energy Conversion Engineering Conference, Providence, RI, 2004, paper AIAA-2004-5557, 8 pp.

${ }^{3}$ Colozza, A.J., Heller, R.S., Wong, W.A. and Hepp, A.F., "Solar Energy Systems For Lunar Oxygen Generation," NASA/ TM-2010-216219, 2010.

${ }^{4}$ Papike, J., Taylor, L., and Simon, S., "Lunar Minerals," in Lunar Sourcebook, edited by G. Heiken, D. Vaniman, and B.M. French, Cambridge University Press, Cambridge, England, UK, 1991, pp. 121-181.

${ }^{5}$ Taylor, L.A., and Carrier, W.D., III, "Oxygen Production on the Moon; An Overview and Evaluation," in Resources of Near-Earth Space, edited by J. Lewis, M.S. Matthews, and M.L. Guerrieri, University of Arizona Press, Tuscon, AZ, 1993, pp. 69-108.

${ }^{6}$ Hepp, A.F., Linne, D.L., Landis, G.A., Wadel, M.F., and Colvin, J.E., "Production and Use of Metals and Oxygen for Lunar Propulsion," J. Propul. Power, Vol. 10, No. 6, 1994, pp. 834-840.

${ }^{7}$ Macosko, R.P., Colozza, A.J., Castle, C., and Sacksteder, K., "Solar Concentrator Concept For Providing Direct Solar Energy For Oxygen Production At The Lunar South Pole," $48^{\text {th }}$ Aerospace Sciences Meeting, Orlando, FL, 2010, paper AIAA2010-1167.

${ }^{8}$ Rabl, A., O'Gallagher, J., and Winston, R., "Design And Test Of Non-Evacuated Solar Collectors With Compound

Parabolic Concentrators," Solar Energy, Vol. 25, no. 4, 1980, p. 335-351.

${ }^{9}$ Winston, R., "Dielectric Compound Parabolic Concentrators," Applied Optics, Vol. 15, No. 2, 1976, pp. $291-292$.

${ }^{10}$ Fikes, J.C., Howell, J.T., Gerrish, H.P., and Patrick, S.L., "Solar Concentrator Demonstrator For Lunar Regolith

Processing," $59^{\text {th }}$ International Astronautical Congress, Glasglow, Scotland, UK, 2008, paper IAC-C3.2.5.

${ }^{11}$ O'Neill, M.J., and Piszczor, M.F., "Inflatable Lenses for Space Photovoltaic Concentrator Arrays," Proceedings of the $26^{\text {th }}$ IEEE Photovoltaic Specialists Conference, IEEE, New York City, 1997, pp. 853-856.

${ }^{12}$ Gardner, J.P. et al., "The James Webb Space Telescope," Space Science Reviews, Vol. 123, Nos. 1-4, 2006, pp. 485-606.

${ }^{13}$ Nakamura, T., and Smith, B.K., "Solar Thermal System For Oxygen Production From Lunar Regolith Ground-based

Demonstration System,” AIAA Space 2009 Conference, Pasadema, CA, 2009, paper AIAA-2009-6509.

${ }^{14}$ Shaltens, R.K., and Boyle, R.V., "Initial Results From The Solar Dynamic (SD) Ground Test Demonstration (GTD) Project At NASA Lewis," NASA TM-107004, 1995.

${ }^{15}$ Wegeng, R.S. et al., "Thermal Wadis In Support Of Lunar Science \& Exploration," $6{ }^{\text {th }}$ International Energy Conversion Engineering Conference, Cleveland, OH, 2008, paper AIAA-2008-5632.

${ }^{16}$ Welford, W.T., and Winston, R., High Collection Nonimaging Optics, Academic Press, San Diego, CA, 1989, pp. 1-24.

${ }^{17}$ Wong, W.A., "Refractive Secondary Solar Concentrator Demonstrated High-Temperature Operation," in 2001 NASA Glenn Research Center Research and Technology Report, NASA/TM-2002-211333, 2002, pp. 74-76.

${ }^{18}$ Colozza, A.J., "Power System Mass Analysis For Hydrogen Reduction Oxygen Production On The Lunar Surface," NASA/CR-2009-21550, 2009.

${ }^{19}$ Briggs, R.A., and Sacco, A., Jr., "Hydrogen reduction mechanisms of ilmenite between 823 and 1353 K," J. Mater. Res., Vol. 6, No. 3, 1991, pp. 574-584.

${ }^{20}$ Gustafson, R.J., White, B.C., and Fidler, M.J., "Demonstrating Lunar Oxygen Production with the Carbothermal Regolith Reduction Process," $47^{\text {th }}$ Aerospace Sciences Meeting, Orlando, FL, 2009, paper AIAA-2009-663.

${ }^{21}$ Jenkins, C.H.M. (ed.), Gossamer Spacecraft: Membrane And Inflatable Structures Technology For Space Applications, Volume 191, Progress in Astronautics and Aeronautics, AIAA, Reston, VA, 2001, pp. 281-320.

${ }^{22}$ Heath, A.R., Jr., and Hoffman, E.L., "Review Of Solar Concentrator Technology," Proceedings of the $1^{\text {st }}$ Intersociety

Energy Conversion Engineering Conference, AIAA, Washington, D.C., 1966, pp. 231-237.

${ }^{23}$ Riihelä, D., Ritala, M., Matero, R., and Leskelä, M., "Introducing Atomic Layer Epitaxy For The Deposition Of Optical

Thin Films," Thin Solid Films, Vol. 289, Nos. 1-2, 1996, pp. 250-255.

${ }^{24}$ James, A.M., and Lord, M.P., Macmillan's Chemical And Physical Data, Macmillan, London, UK, 1992.

${ }^{25}$ Laby, T.H., Tables Of Physical And Chemical Constants, URL: http://www.kayelaby.npl.co.uk/toc/.

${ }^{26}$ Lide, D.R. (ed.), Handbook Of Chemistry And Physics, 78th ed., CRC Press LLC, Boca Raton, FL, 1997.

${ }^{27}$ Dean, J.A., Lange's Handbook Of Chemistry, 15th ed., McGraw-Hill, New York, NY, 1998.

${ }^{28}$ Harrison, R.D. (ed.), Nuffield Advanced Science Book of Data (Longman, London, UK, 1972.

${ }^{29}$ Samsonov, G.V. (ed.), Handbook Of The Physicochemical Properties of the Elements (IFI/Plenum, New York, NY, 1968

${ }^{30}$ Gaier, J.R., "The Effects of Lunar Dust on EVA Systems During the Apollo Missions," NASA/TM-2005-213610, 2005.

${ }^{31}$ Stubbs, T.J., Vondrak, R.R., and Farrell, W.M., "Impact of Dust on Lunar Exploration," in Workshop on Dust in Planetary Systems (ESA SP-643), Kauai, Hawaii, 2005, Krueger, H. and Graps, A., ed., ESA Publications, Noordwijk, The Netherlands, 2007, pp.239-243.

${ }^{32}$ Katzan, C.M., and Edwards, J.L., "Lunar Dust Transport and Potential Interactions with Power System Components," NASA CR-4404, 1991.

${ }^{33}$ Nakamura, T., and Senior, C.L., "Solar Thermal Power For Lunar Materials Processing,” J. Aerospace Eng., Vol. 21, No. 2, 2008, pp. 91-101

${ }^{34}$ Naval Research Laboratory, "The Clementine Mission,” URL: http://www.cmf.nrl.navy.mil/clementine/. 
${ }^{35}$ Colozza, A.J., and Wong, W.A., "Evaluation Of A Stirling Solar Dynamic System For Lunar Oxygen Production," NASA/TM-2006-214360, 2006.

${ }^{36}$ Angel, J.R.P., "Buyer's Guide To Telescopes At The Best Sites: Dome A, L2, And Shackleton Rim,” Optical, Infrared, and MIllimeter Space Telescopes, SPIE Proceedings, Volume 5487, 2004, pp. 1-12.

${ }^{37}$ Hartenstine, J.R., Anderson, W.G., Walker, K.L., and Ellis, M.C., "Heat Pipe Solar Receiver For Oxygen Production of Lunar Regolith," Space, Propulsion and Energy Sciences International Forum: SPESIF-2009, AIP Conference Proceedings, Volume 1103, 2009, pp. 105-113.

${ }^{38}$ Wynne, C.G., "Ritchey Chrétien Telescopes And Extended Field Systems," The Astrophysical Journal, Vol. 152, 1968, pp. 675-694.

${ }^{39}$ Pitz-Paal, R., "High Temperature Solar Concentrators," Solar Energy Conversion and Photoenergy Systems, edited by J. Blanco and S. Malato, Encyclopedia of Life Support Systems (EOLSS), EOLSS Publications, Mississauga, ON, Canada, 2007, URL: http://www.eolss.net/.

${ }^{40}$ Colozza, A.J., "Analysis of Lunar Regolith Thermal Energy Storage,” NASA-CR 189073, 1991.

${ }^{41}$ Edmund Optics, URL: www.edmundoptics.com. 